\title{
The rough favourable pressure gradient turbulent boundary layer
}

\author{
RAÚL BA YOÁN CAL ${ }^{1} \dagger$, BRIAN BRZEK ${ }^{2}$, \\ T. GUNNAR JOHANSSON ${ }^{3}$ AND LUCIANO CASTILLO
}

${ }^{1}$ Department of Mechanical and Materials Engineering, Portland State University, Portland, OR 97207-0751, USA

${ }^{2}$ Department of Mechanical, Aerospace and Nuclear Engineering, Rensselaer Polytechnic Institute, Troy, NY 12180, USA

${ }^{3}$ Department of Applied Mechanics, Chalmers University of Technology, SE-41296 Gothenburg, Sweden

(Received 28 January 2009; revised 3 August 2009; accepted 4 August 2009; first published online 25 November 2009)

Laser Doppler anemometry measurements of the mean velocity and Reynolds stresses are carried out for a rough-surface favourable pressure gradient turbulent boundary layer. The experimental data is compared with smooth favourable pressure gradient and rough zero-pressure gradient data. The velocity and Reynolds stress profiles are normalized using various scalings such as the friction velocity and free stream velocity. In the velocity profiles, the effects of roughness are removed when using the friction velocity. The effects of pressure gradient are not absorbed. When using the free stream velocity, the scaling is more effective absorbing the pressure gradient effects. However, the effects of roughness are almost removed, while the effects of pressure gradient are still observed on the outer flow, when the mean deficit velocity profiles are normalized by the $U_{\infty} \delta_{*} / \delta$ scaling. Furthermore, when scaled with $U_{\infty}^{2}$, the $\left\langle u^{2}\right\rangle$ component of the Reynolds stress augments due to the rough surface despite the imposed favourable pressure gradient; when using the friction velocity scaling $u_{*}^{2}$, it is dampened. It becomes 'flatter' in the inner region mainly due to the rough surface, which destroys the coherent structures of the flow and promotes isotropy. Similarly, the pressure gradient imposed on the flow decreases the magnitude of the Reynolds stress profiles especially on the $\left\langle v^{2}\right\rangle$ and $-\langle u v\rangle$ components for the $u_{*}^{2}$ or $U_{\infty}^{2}$ scaling. These effects are reflected in the boundary layer parameter $\delta_{*} / \delta$, which increase due to roughness, but decrease due to the favourable pressure gradient. Additionally, the pressure parameter $\Lambda$ found not to be in equilibrium, describes the development of the turbulent boundary layer, with no influence of the roughness linked to this parameter. These measurements are the first with an extensive number of downstream locations (11). This makes it possible to compute the required $x$-dependence for the production term and the wall shear stress from the full integrated boundary layer equation. The finding indicates that the skin friction coefficient depends on the favourable pressure gradient condition and surface roughness.

Key words: boundary layers 


\section{Introduction}

The effects of roughness and favourable pressure gradient (FPG) on turbulent boundary layers have been studied extensively, but separately. The applications of these types of flows extend to nozzle design, drag reduction applications, ship hull design, atmospheric boundary layers and space technology to name a few. These combined effects produce a more realistic picture of flows which happen in many engineering applications, particularly at high Reynolds numbers where roughness becomes important.

Up until now, only one study combining the effects of roughness and FPG exists in the literature. Coleman, Moffat $\&$ Kays (1977) used hot-wire anemometry to measure the velocity field in three downstream locations in a turbulent boundary layer subject to both roughness and FPG. The roughness used was an arrangement of copper spheres. The external FPG was created using a flexible Plexiglas at the upper wall of the wind tunnel. The study focused primarily on equilibrium boundary layers as proposed by Clauser (1954). The skin friction was acquired using the momentum integral equation without the inclusion of the streamwise gradients of the $\left\langle u^{2}\right\rangle$ and $\left\langle v^{2}\right\rangle$ terms. A pressure gradient parameter for rough walls was also suggested as

$$
K_{r}=\frac{r}{U_{\infty}} \frac{\mathrm{d} U_{\infty}}{\mathrm{d} x},
$$

where $r$ is the radius of the spheres. Another explored effect was the use of blowing on the surface. A variety of velocity scales and length scales was used to normalize the profiles. A change in the location of the peaks for the $\left\langle u^{2}\right\rangle$ component when scaled with $u_{*}^{2}$ occurred with accelerated and non-accelerated data. When scaled with $U_{\infty}^{2}$, the peaks coincide. Unfortunately, these measurements lacked adequate near-wall resolution, especially for the Reynolds stresses. The integral parameters all become constants in these layers. The flow becomes less isotropic near the wall as the FPG is increased, as is also shown in this study. In addition, the viscous sublayer is destroyed by the roughness elements. Contrary to Coleman et al. (1977), the current investigation does not attempt to achieve an equilibrium state as proposed by Clauser, where the pressure parameter,

$$
\beta=\frac{\delta_{*}}{\rho u_{*}^{2}} \frac{\mathrm{d} P_{\infty}}{\mathrm{d} x},
$$

equals a constant and the velocity deficit profiles,

$$
\frac{U_{\infty}-U}{u_{*}}=f\left(\frac{y}{\delta}\right)
$$

are independent of the streamwise direction.

Rough surfaces were initially studied by Nikuradse (1932) on pipe flows and different roughness regimes were defined. The regimes were based on the non-dimensional parameter which contains the equivalent sand grain roughness $k_{e q}$ given as

$$
k^{+}=\frac{k_{e q} u_{*}}{v},
$$

where $u_{*}$ is the friction velocity and $v$ is the kinematic viscosity. The ranges for the roughness regimes were classified as hydraulically smooth (i.e. $k^{+}<5$ ), transitionally rough (i.e. $\left.5<k^{+}<70\right)$ and fully rough $\left(k^{+}>70\right)$. Both the equivalent sand grain roughness and the friction velocity are quantities which are not easy to compute or measure. 
The effects of roughness on the inner and outer layers of a zero pressure gradient (ZPG) flow were studied by Krogstad, Antonia \& Browne (1992) by using a roughness consisting of a mesh-screen. These changes were observed on the velocity profiles, and the outer 'wake' region was found to be larger than on smooth walls. A quadrant analysis of the Reynolds stresses was also performed, and the effect of this particular roughness on sweeps and ejections was studied. Bergstrom, Kotey \& Tachie (2002) performed experiments on a rough turbulent boundary layer by studying effects such as roughness conditions and wind-tunnel speed. The velocity profiles were normalized using the friction velocity obtained using the Clauser chart and the free stream velocity. Both normalizations showed an influence due to the surface roughness. Furthermore, Krogstad \& Antonia (1994), Shafi \& Antonia (1995), Krogstad \& Antonia (1999), Tachie, Bergstrom \& Balachandar (2000) and Akinlade et al. (2004) found that the mean velocity defect in the outer layer and turbulence structure differs among the different types of rough surfaces, as well as from that in a smooth wall boundary layer. This contradicts the wall similarity hypothesis and suggests that boundary layers over different rough walls will pose a significant challenge for turbulence modellers. Brzek et al. (2007) studied the effects of sandgrain roughness in ZPG turbulent boundary layers, including all the roughness regimes over a significant Reynolds number range. Self-similar solutions were obtained for fixed sets of experimental conditions, regardless of the scaling used for both mean velocity and Reynolds stress profiles. However, differences in roughness geometry resulted in differences in the Reynolds stress profiles. This study suggests that the similarity hypothesis is a restricted condition that holds for few flows. Among the conditions are the ratio of $k / \delta<0.02$ and Reynolds number, $R e_{\theta}>10,000$ as shown by Jiménez (2004). Studies performed by Perry \& Li (1990), Jiménez (2004), Bakken et al. (2005) and Schultz \& Flack (2005) satisfy such criteria. However, recent studies done by Flack, Schultz \& Shapiro (2005), Castro (2007) and Flack, Schultz \& Connelly (2007) suggest that outer similarity still holds at a higher value of the $k / \delta$ ratio.

Other types of roughness elements with different geometrical properties have been used. As stated by Leonardi et al. (2003), height, shape, spacing and bluntness (rods, mesh and sand grains) have a profound effect on the properties of the flow in a channel. The geometric properties of the roughness elements are not used directly in the characterization of sand grain roughness with the exception of the roughness height. The characteristics of such surfaces were studied in detail by Schultz (2002). Three-dimensional measurements of a painted surface and sand grain type surfaces were acquired, but the one-dimensional roughness height based on Nikuradse's sand grain height was used to describe the surface. This is based on the roughness function $\Delta U^{+}\left(k^{+}\right)$instead of the geometry of the surface, which represents the downward shift due to the roughness on the velocity profiles.

The $\Delta U^{+}\left(k^{+}\right)$function is a quantity which is part of the classical law of the wall for rough turbulent boundary layers given as

$$
\frac{U}{u_{*}}=\frac{1}{\kappa} \ln (y+\epsilon)^{+}+B+2 \omega\left(\frac{y}{\delta}\right) \frac{\Pi}{\kappa}-\Delta U^{+},
$$

where $\kappa$ is the von Kármán constant, $\epsilon$ is the shift for the adjustment of the wall distance, $B$ is the smooth wall intercept, $2 \omega(y / \delta) \Pi / \kappa$ is the wake function proposed by Coles (1962) and $\Pi$ is the wake parameter. This is used in the modified Clauser (1954) chart method to determine the friction velocity $u_{*}$. Moreover, the friction velocity is a quantity which can be obtained indirectly from methods like the modified Clauser chart by Clauser (1954) or the fully integrated boundary layer equation. The main difference between the commonly used chart on smooth flows and rough flows is 
the roughness function $\Delta U^{+}\left(k^{+}\right)$. Generally, the calculation of the proper value of the skin friction is a major challenge in turbulent boundary layers, particularly in rough surfaces. The major drawback of the modified Clauser chart method is the dependence on several different parameters, $\epsilon, u_{*}, k_{e q}$, as well as the wake function, which are necessary to determine the skin friction. In addition, a consequential problem that occurs as the strength of the FPG increases, is that a breakdown of the logarithmic region as found by Warnack \& Fernholz (1998). This makes the problem of acquiring the skin friction even more complex on rough FPG turbulent boundary layers. Ligrani \& Moffat (1986) used the integral momentum equation technique, while Karlsson (1980) used a skin friction balance. The implementation of the integrated momentum equation for the determination of the wall shear stress is not possible in many cases. Most experiments do not possess the required number of streamwise locations ( $x$-dependence) of the flow field to calculate the streamwise gradients, as shown by Newhall et al. (2006) and Cal et al. (2008); in some cases the flow is not two-dimensional.

Launder (1964) proposed an acceleration parameter for FPG flows given as

$$
K=\frac{v}{U_{\infty}^{2}} \frac{\mathrm{d} U_{\infty}}{\mathrm{d} x} .
$$

FPG flows were first studied by Ludweig \& Tillman (1950), Herring \& Norbury (1966) and Kline et al. (1967). However, only mean velocity data was documented. Other experiments were also carried out on strong FPG flows by Launder (1964), Patel \& Head (1968) and Narasimha \& Sreenivasan (1973). A review of many of these flows was provided by Sreenivasan (1982). Recent experiments by Fernholz \& Warnack (1998) and Ichimiya, Nakamura \& Yamashita (1998), Warnack \& Fernholz (1998), Jones, Marusic \& Perry (2001), Mukund et al. (2006) and Cal \& Castillo (2008) explored the phenomenon of quasi-laminarization. A decrease in the Reynolds stresses and eventual decrease in the skin friction were shown in studies carried out by Piomelli, Balaras \& Pascarelli (2000), Radhakrishnana, Keating \& Piomelli (2006) and Cal \& Castillo (2008).

The effects of roughness and FPG have yet to be reported in the literature, especially regarding near-wall measurements of Reynolds stresses. Therefore, Laser Doppler anemometry (LDA) measurements of a rough turbulent boundary layer, subject to an FPG provide insight on the interaction between the pressure gradient and the surface roughness. These results are a follow-up to the paper of Cal et al. (2008), which document Reynolds number effects on a weaker pressure gradient condition on the flow field. A unique feature of this experiment is the 11 consecutive streamwise locations where the velocity and Reynolds stress fields were measured, which yielded a total of 33 profiles. Therefore, the $x$-dependence of the flow is known, and thus, gradients of the streamwise component could be calculated (i.e. production, $\mathrm{d} U / \mathrm{d} x$, $\tau_{w} / \rho$, etc.). The mean velocity deficit and Reynolds stress profiles are herein analysed using different scalings that highlight the behaviour caused by the imposed surface roughness and the external FPG. In addition, the development of the boundary layer is observed along with the boundary layer parameters (i.e. ratio of $\delta_{*} / \delta$, the boundary layer growth and the pressure parameter $\Lambda$ ).

\section{Outer scalings for velocity and Reynolds stress}

Different scales are used to assess the effects due to the FPG and surface roughness on the turbulent boundary layer. Traditionally, the friction velocity $u_{*}$ is used for scaling the mean velocity deficit and the Reynolds stresses. Other scalings have been 


$\begin{array}{cc}\text { Velocity deficit scalings } & \text { Reynolds stress scalings } \\ \left(U_{\infty}-U\right) & \left(\left\langle u^{2}\right\rangle,\left\langle v^{2}\right\rangle \&-\langle u v\rangle\right) \\ u_{*} & u_{*}^{2} \\ U_{\infty} & U_{\infty}^{2} \\ U_{\infty} \delta_{*} / \delta & \end{array}$

TABLE 1. Scalings employed for velocity and Reynolds stress profiles.

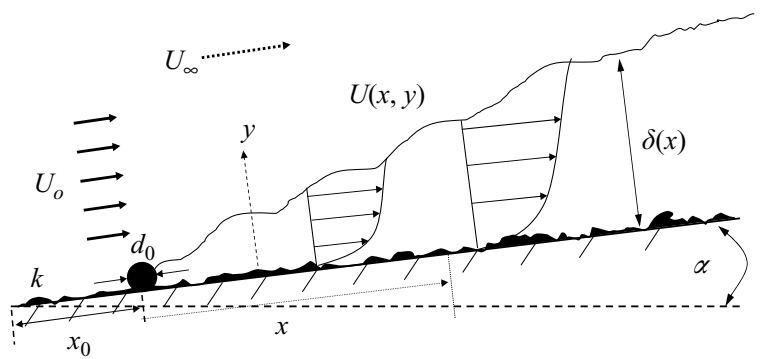

FIGURE 1. Depiction of the rough turbulent boundary layer subject to a favorable pressure gradient.

proposed as in Degraaff \& Eaton (2000). For example, following the similarity analysis proposed by Castillo \& George (2001) on pressure gradient flows, the scales for the outer flow are obtained without assuming anything a priori (also for George (1994) and George \& Castillo (1997) for ZPG flows). These scalings are tabulated on table 1. For comparison purposes, the scaling $U_{\infty} \delta_{*} / \delta$ is also employed for the velocity deficit profiles. The scaling has been able to collapse the effects of upstream conditions and surface roughness into a single functional relationship as seen by Castillo \& Walker (2002) and Castillo et al. (2004a).

Similarly, the Reynolds stresses are an additional set of dependent variables that may be affected by surface roughness and pressure gradients. Thus, Reynolds stresses may provide a source of information to improve the understanding of how boundary layers are modified by these external conditions (i.e. the energy exchange between the terms and components). As shown in table 1, the scalings used for the Reynolds stresses are the friction velocity $u_{*}^{2}$ and the free stream velocity $U_{\infty}^{2}$. By matching the Reynolds stress in inner and outer variables in the limit as the Reynolds number approaches infinity, it follows that $U_{\infty}^{2} \mathrm{~d} \delta / \mathrm{d} x \sim u_{*}^{2}$ for ZPG flows as proposed by George \& Castillo (1997). Consequently, the scale for the pressure-strain rate, diffusion and dissipation terms are characterized by a mixed scaling between $u_{*}$ and $U_{\infty}$.

\section{The experiment}

The wind-tunnel facility used for these experiments is the L2 wind-tunnel at the Department of Applied Mechanics at Chalmers University of Technology. The facility is a conventional closed-loop design, equipped with turning vanes in all four corners with honeycombs and screens. The contraction ratio is 5.6:1, and the free stream turbulence level is approximately $0.1 \%$. The test section is $3 \mathrm{~m}$ long, $1.8 \mathrm{~m}$ wide and $1.25 \mathrm{~m}$ high.

To create an FPG, a specially manufactured aluminum plate is mounted vertically at an angle $\alpha$, as seen in figure 1. The plate has dimensions of $2.5 \mathrm{~m}$ long, $1.25 \mathrm{~m}$ 


$\begin{array}{clcc}\text { Case } & \text { Condition } & \text { Viscous length scale }(\mu \mathrm{m}) & \text { Probe diameter/viscous length scale } \\ 1 & \text { Smooth FPG } & 20 & 2.9 \\ 2 & \text { Rough FPG } & 15 & 4.3 \\ 3 & \text { Rough ZPG } & 30 & 1.9\end{array}$

TABLE 2. Spatial resolution for the present experiments with upstream wind tunnel speed of $10 \mathrm{~m} \mathrm{~s}^{-1}$.

wide and $5 \mathrm{~mm}$ thick. The nose of the plate is placed $200 \mathrm{~mm}$ downstream from the test section entrance. In both the smooth and rough FPG experiments, the plate is kept at an angle of $15^{\circ}$ to the oncoming air stream. In the ZPG experiment, the plate is parallel with the air stream. Moreover, for these FPG and ZPG experiments, the boundary layer is disturbed with a cylindrical trip-wire of $2 \mathrm{~mm}$ in diameter and positioned $150 \mathrm{~mm}$ downstream from the leading edge. The upstream wind-tunnel speed $U_{o}$, trip-wire size $d_{o}$ and position $x_{o}$ define the upstream conditions and are denoted in figure 1 . Measurements are performed in 11 consecutive downstream locations, moving from $1.30 \mathrm{~m}$ up to $2.30 \mathrm{~m}$ from the leading edge of the plate. In the ZPG case, the local free stream speed is constant, equal to $U_{\infty}$. In the FPG cases, the local free stream speed increases, and reaches $13 \mathrm{~m} \mathrm{~s}^{-1}$ at the first measuring station and $19 \mathrm{~m} \mathrm{~s}^{-1}$ at the last one.

The velocity field is measured using a two component LDA system. The system consists of a nearly vertical probe emitting four beams of light, two blue and two green, from an Argon-ion laser. A reduction of the control volume size is achieved by using two expanders with an expansion ratio of 1.94:1 and one focusing beam expander with an expansion ratio of 1.55:1. The focusing beam expander has a focal length of $1200 \mathrm{~mm}$. Using a back-scatter arrangement, the measuring control volume is nearly prolate ellipsoidal with a diameter of about $58 \mu \mathrm{m}$ and a length of about $600 \mu \mathrm{m}$. Therefore, the smallest scales of the flow are resolved or nearly resolved depending on the local flow conditions. The spatial resolution in the wall-normal direction is shown in table 2, which includes the viscous length scale and the probe volume diameter to viscous length scale ratio. The best resolution is achieved for the rough ZPG data with the larger viscous scale, while the rough FPG data is a factor of 2 smaller than the rough ZPG. The uncertainties for the mean velocity and Reynolds stresses are determined based on the number of samples, which is greatly impacted by the wall proximity. For instance, for the few data points near the wall, low data rates are observed. On average, 2000-6000 samples are obtained per point during a $200 \mathrm{~s}$ sampling time interval. It is important to note that the sampling rate varied considerably from one point to another. For the rough ZPG set the error in the mean velocity measurements is less than $1 \%$; in the fluctuating quantities it is estimated to be between $2-3 \%$, while the joint moment is between $4-6 \%$. For the smooth and rough FPG case, the error is estimated to be less than $1 \%$ in the mean, $5 \%$ for the fluctuations and between 6-9\% for the Reynolds shear stress. Furthermore, the closest measurements to the wall for the rough-surface data are obtained at about $y^{+} \approx 25$ and about $y^{+} \approx 5$ for the smooth case.

A SAFEX fog generator 2010 (110 VAC) is used to induce droplets which follow the flow and do not interfere with its motion. This generator is placed in the diffuser part of the wind tunnel. The fluid used to create the particles is SAFEX standard fog fluid inside Nebelfluid normal power mix. The droplets possess a mean droplet size of $1 \mu \mathrm{m}$. The typical fog durability in confined areas with this standard fluid is 


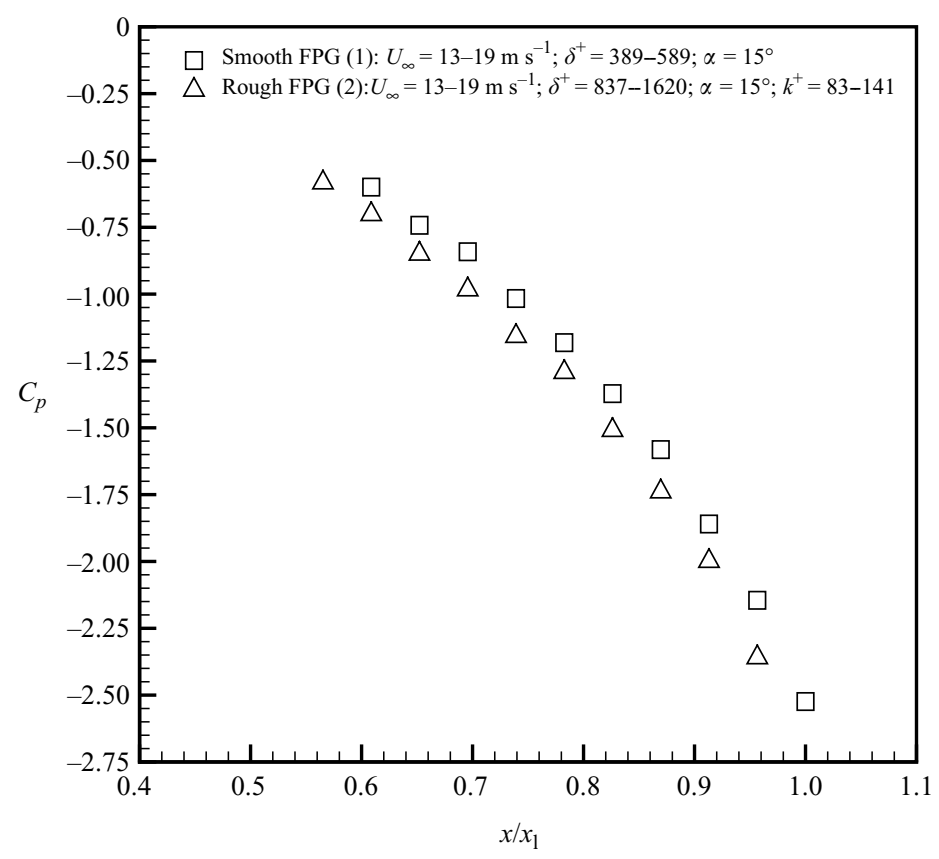

FIGURE 2. Pressure distribution for FPG data.

10-30 min. The flow is seeded periodically to obtain good signal quality, consistent data rates and data validation.

Due to the randomness of the surface, the positioning of the control volume relative to the wall is challenging. Therefore, a smooth rectangular piece of glass with a constant thickness of $9.75 \mathrm{~mm}$ is placed on top of the surface. The probe is then traversed until the control volume touches the flat surface of the glass and then moved back towards the wall the thickness of the glass. This location is then recognized as the zero position of the surface. A correction for the location of the virtual origin, known as $\epsilon$, is performed using the method of Perry \& Li (1990). Further details about the experiment on the smooth FPG flow are found in Cal, Johansson \& Castillo (2006) and on rough ZPG flows by Brzek et al. (2007) and Brzek et al. (2008).

\subsection{The external conditions}

Each experiment provides the isolation of the external studied condition to observe the influence of the particular effect on the downstream flow. Three of the external conditions are fixed for all three cases: (i) the upstream wind-tunnel speed $U_{o}$, (ii) tripwire size $d_{o}$ and (iii) trip-wire location $x_{o}$. These conditions have values of $10 \mathrm{~m} \mathrm{~s}^{-1}$, $2 \mathrm{~mm}$ and $150 \mathrm{~mm}$, respectively. These new measurements take into account a strong FPG given by the angle of the plate, $\alpha=15^{\circ}$. The similarities between the two pressure gradient cases with smooth and rough surfaces are observed in figure 2, where the pressure distribution $C_{p}$ is plotted against the normalized streamwise position. A comparable magnitude and trend for both FPG cases is observed, thus allowing an accurate comparison of these data sets.

Table 3 shows the case number in the first column. The second column corresponds to the angle of the plate $\alpha$, which determines the strength of the external pressure gradient. Given that FPG flows are classically quantified using the acceleration 


\begin{tabular}{ccccccccl}
\hline & & & & & & & & \\
Case & $\alpha(\operatorname{deg})$ & $K\left(\times 10^{-7}\right)$ & $k^{+}$ & $\bar{k}$ & $k_{s}^{+}$ & $\operatorname{Re}_{\theta}$ & $\delta^{+}$ & $C_{f} \times 10^{-3}$ \\
1 & 15 & $4.11-4.60$ & 0 & 0 & 0 & $1118-1552$ & $389-589$ & $3.71-4.51$ \\
2 & 15 & $3.77-4.71$ & $83-141$ & $0.087-0.099$ & $103-248$ & $2066-3399$ & $837-1620$ & $8.40-10.30$ \\
3 & 0 & $0.027-0.028$ & $49-54$ & $0.035-0.053$ & $77-92$ & $3309-4927$ & $1040-1440$ & $4.84-5.72$
\end{tabular}

TABLE 3. External conditions and dependent variables for the experimental turbulent boundary layers.

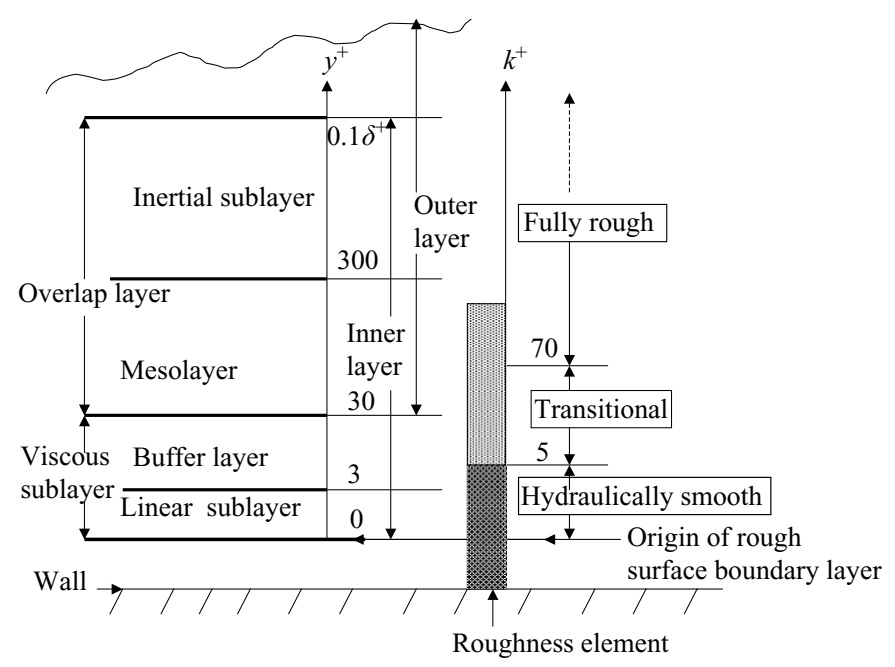

FIGURE 3. Rough boundary layer regimes, Seo (2003).

parameter, $K=v / U_{\infty}^{2} \mathrm{~d} U_{\infty} / \mathrm{d} x$, this parameter is tabulated in the third column. The value for the ZPG flow is very small, thus negligible as expected. Next, the roughness parameter $k^{+}$range is shown, which includes all three regimes: hydraulically smooth, transitional and fully rough. For instance, Case 1 is in the hydraulically smooth regime, Case 2 is in the fully rough regime, and finally, Case 3 is in the transitionally rough regime. These regimes are appreciated along with the counterpart of the boundary layer regions shown in figure 3 . Following, $\bar{k}$ is shown for the different cases where $\bar{k}=k / \delta$ is the roughness height to boundary layer thickness ratio. The $\bar{k}$ parameter has been employed by Jiménez (2004) to confirm when the Townsend (1956) similarity hypothesis is satisfied. The hypothesis is valid for values of this parameter lower than 0.02 and for high Reynolds numbers, $R e_{\theta}>10000$, where the overlap exists as observed in George \& Castillo (1997); for values greater than $\bar{k}>0.02$, there is a breakdown of the hypothesis in ZPG flows. During this breakdown, roughness influences the outer layer. Also, the values of $k_{s}^{+}$are given through the equation $\Delta U^{+}=1 / 0.41 \ln \left(k_{s}^{+}\right)-3.2$ following the study of Raupach, Antonia \& Rajagopalan (1991) for the fully rough regime. The values of $k_{s}^{+}$vary from 100-250 as the flow develops over the plate. Moreover, the values for the rough ZPG flow were determined using the technique of Ligrani \& Moffat (1986) since this flow falls in the transitionally rough regime. Furthermore, the Reynolds numbers, $R e_{\theta}$ and $\delta^{+}$, are represented in the next two columns. These ranges do not suffice to satisfy the Townsend hypothesis as given by Jiménez (2004). Finally, the range for the skin friction coefficient $C_{f}$ in the last column is determined from the full integrated boundary layer equation 
(defined in (3.1)). These last three quantities are dependent variables and should not be considered as external or upstream conditions.

Given the FPG, a fully rough regime is achieved with the 24-grit sand paper. As a result, the range of $k^{+}$is from $0-141$, and the range of Reynolds number based on the momentum thickness, $R e_{\theta}$, is from $1118 \leqslant R e_{\theta} \leqslant 4927$. These regimes are consistent with the different layers of the boundary layer in terms of inner and outer flow, which are herein described as in Seo (2003) and shown in figure 3. In the proximity of the wall, the linear subrange is dominated by the viscous terms and extends up to $(y+\epsilon)^{+} \approx 3$. A combination of the viscous stress and Reynolds stress compose the buffer layer $\left(3<(y+\epsilon)^{+}<30\right)$. The overlap region is then comprised of the mesolayer $30<y^{+}<300$ and the inertial sublayer between $300<(y+\epsilon)^{+}<0.1 \delta^{+}$. An inertial sublayer for the reported measurements does not exist due to the low ranges of Reynolds numbers. An inertial sublayer begins to exist at about $R e_{\theta} \approx 10,000$; thus the overlap region is characterized mainly by the mesolayer, which for this lowReynolds-number data is about $25 \%$ of the boundary layer as pointed out by George \& Castillo (1997) and Brzek et al. (2008).

The rough surface considered in these experiments is a 24-grit aluminum oxide open-coated sand paper. The sheet is attached to the entire length of the aluminum plate and wrapped around the leading edge. The sheet is $0.6 \mathrm{~m}$ wide and is placed in the centre of the $1.25 \mathrm{~m}$ high plate. Double-sided tape covering the majority of the surface is used to attach the sand paper to the aluminum plate.

A single hydraulically relevant roughness height $k$ is a difficult parameter to determine from purely geometrical information for complex surfaces. Therefore, the roughness height $k$ used in this investigation is somewhat arbitrarily chosen as the average of the five highest peaks and five deepest valleys, $S_{z}=1.522 \mathrm{~mm}$, where according to Bradshaw (2000), the contribution of the larger peaks is significant compared to the smaller peaks. For more details about the surface refer to Cal et al. (2008). Moreover, the roughness function $\Delta U^{+}$is not used since it depends on the assumption that the overlap for rough surfaces subject to an FPG can be characterized by a logarithmic function. For relatively strong favourable pressure gradients, it is known that the log-law fails to properly describe the overlap region, as described by Fernholz \& Warnack (1998).

\subsection{The boundary layer parameters}

The ratio of $\delta_{*} / \delta$ is the parameter included in the Zagarola and Smits (ZS) scaling (Zagarola \& Smits 1998) and is shown in figure 4(a). This parameter decreases for the smooth FPG case due to the imposed favourable pressure gradient acting on the flow. The change occurs over a limited range of Reynolds numbers. However, notice that for rough surfaces, this parameter is nearly Reynolds number invariant even in the transitionally rough regime. This is caused by the disappearance of the viscous sublayer due to the roughness elements interacting with the inner flow. However, the effects of the rough surface and FPG are opposite to each other.

Figure $4(b)$ shows the plot of $\log \left(U_{\infty} / U_{\infty i}\right)$ versus $\log \left(\delta / \delta_{i}\right)$, where $U_{\infty i}$ and $\delta_{i}$ are the values of the free stream velocity and the boundary layer thickness at the first measurement location, respectively. These values are used to normalize the data. The slope of this curve shows the pressure parameter defined by $\Lambda \equiv$ $-\delta\left(\mathrm{d} U_{\infty} / \mathrm{d} x\right) / U_{\infty} \mathrm{d} \delta / \mathrm{d} x \equiv \delta\left(\mathrm{d} P_{\infty} / \mathrm{d} x\right) / \rho U_{\infty}^{2} \mathrm{~d} \delta / \mathrm{d} x$. If $\Lambda=$ constant, it follows that $\delta \sim$ $U_{\infty}^{-1 / \Lambda}$. For these data sets, linear behaviour is not observed and $\Lambda \neq$ constant; instead, a development is observed as the flow evolves downstream. Therefore, these FPG flows, including smooth and rough surfaces, are not in equilibrium since $\Lambda \neq$ 

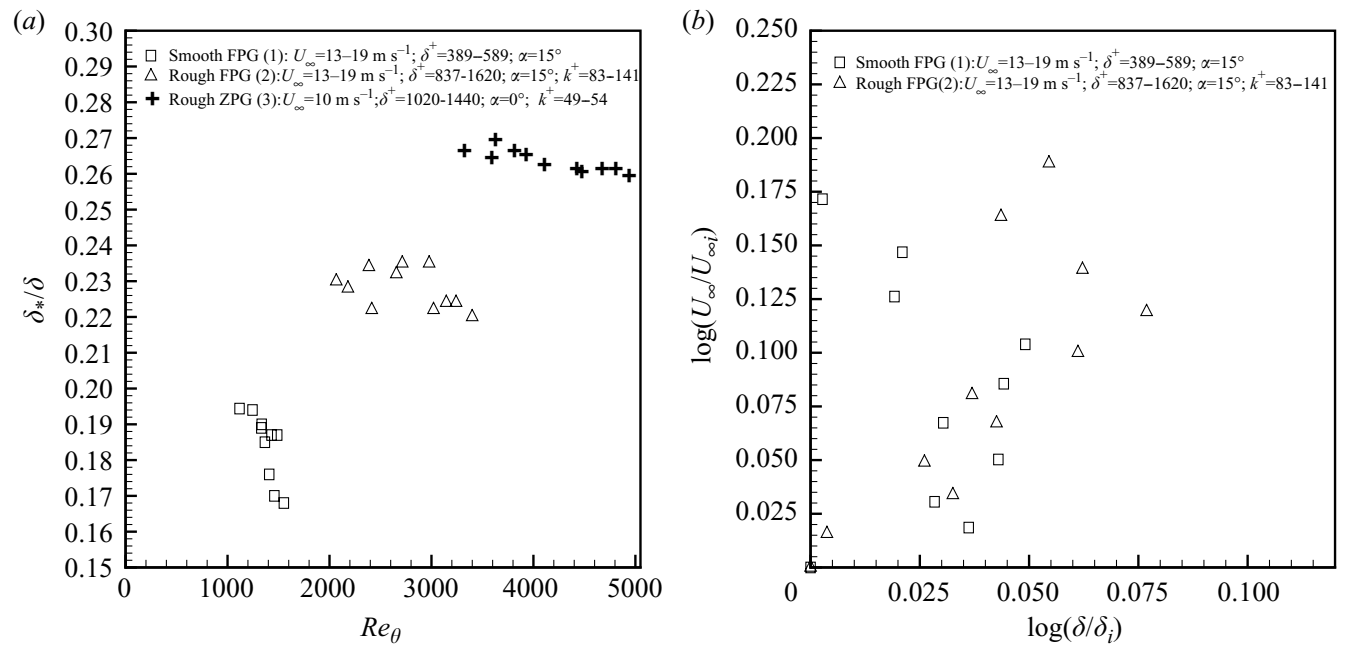

FiguRE 4 . The boundary layer parameters. (a) The boundary layer parameter $\delta_{*} / \delta$. $(b)$ The pressure parameter, $\Lambda$.

constant. The data points begin to move to the right and as the FPG strength increases, the trend shifts to the left with a small decrease in boundary layer thickness. There is no clear evidence of an effect due to roughness on the pressure parameter. In fact, the two data sets are similar in their trend within scatter of the data. Although there is significant scatter in the data, these conclusions then lead to hypothesize that both conditions affect the flow, but that these effects are not necessarily merged together. It is also important to note that the equilibrium condition in this case is different from the condition proposed by Clauser. In the sense of Clauser (1954), an equilibrium flow is one where the mean velocity deficit profiles normalized by the friction velocity are independent of the streamwise direction and $\beta=\left(\delta_{*} / \rho u_{*}^{2}\right) \mathrm{d} P_{\infty} / \mathrm{d} x$ equals a constant. Castillo \& George (2001) showed that few flows satisfy this condition and most flows satisfy the condition of $\Lambda=$ constant. In fact, the Clauser conditions are more restrictive conditions to achieve an equilibrium flow than the pressure parameter, $\Lambda$. The pressure parameter is obtained as a condition through the similarity analysis of the Reynolds averaged Navier-Stokes equations.

\subsection{The boundary layer growth}

The growth of each of the boundary layers is observed in figure 5. The rough FPG data grows faster and thicker than the smooth FPG data. This occurs due to the interaction of the boundary layer with the rough wall. Moreover, the data points for the rough FPG set are less constant than for the rough ZPG data set. Furthermore, the rough ZPG data grows thicker than either one of the FPG cases. The boundary layer thickness also reduces as the external FPG increases. This is due to the acceleration of the boundary layer in the streamwise direction. The classical viewpoint about the influence of the external conditions on the flow and its history are easily contradicted. These results show that the external conditions do affect the boundary layer. In addition, it is appreciated here that each boundary layer reaches its own asymptotic state. Therefore, these boundary layers are dependent on the imposed external conditions and definitively remember how they were created. 


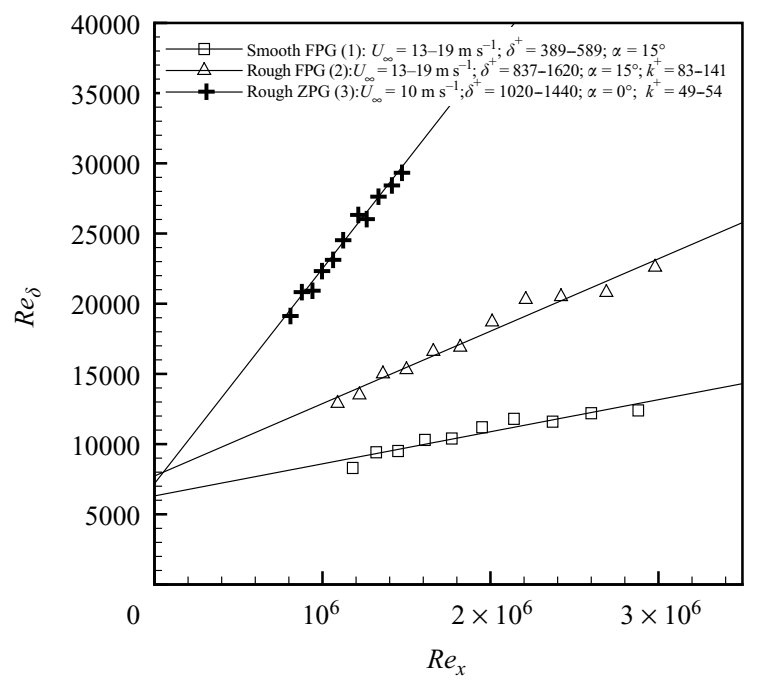

FIGURE 5. The growth of the boundary layers.

\subsection{The wall shear stress}

The integrated boundary layer equation is used to determine the friction velocity as shown in (3.1). To be able to use the fully integrated boundary layer equation, the measured mean velocity and Reynolds stress profiles are necessary in many streamwise locations, as well as, accurate measurements near the wall. With this information, the $x$-derivatives in the boundary layer equation can be computed. Refer to Cal et al. (2008) for more details:

$$
\begin{aligned}
\frac{\tau_{w}}{\rho}= & v \frac{\partial U}{\partial y}-\langle u v\rangle-\int_{0}^{y} \frac{\partial U^{2}}{\partial x} \mathrm{~d} y^{\prime}+U \int_{0}^{y} \frac{\partial U}{\partial x} \mathrm{~d} y^{\prime} \\
& -\int_{0}^{y} \frac{\partial\left\langle u^{2}\right\rangle}{\partial x} \mathrm{~d} y^{\prime}+\int_{0}^{y} \frac{\partial\left\langle v^{2}\right\rangle}{\partial x} \mathrm{~d} y^{\prime}+U_{\infty} \frac{\mathrm{d} U_{\infty}}{\mathrm{d} x} y .
\end{aligned}
$$

In figure $6(a)$, the momentum balance between the terms described above is shown for a rough FPG flow with $U_{\infty}=15 \mathrm{~m} \mathrm{~s}^{-1}, \delta^{+}=1100, \alpha=15^{\circ}$ and $k^{+}=106$. The analysed location is at $1.6 \mathrm{~m}$ from the leading edge since the derivatives at the interior downstream locations are most accurate. The breakdown of the wall shear stress into all the different contributing terms allows for realization of the relative strength of each term in different regions of the boundary layer. Close to the wall $(y / \delta<0.1)$, it is observed that the Reynolds shear stress is the dominant term along with the strong pressure gradient term, where these represent about $80 \%$ and $20 \%$ contributions, respectively. The other terms do not play a significant role. Also, the viscous term is negligible in the inner region, where the high value of the roughness parameter $\left(k^{+}=106\right)$ destroys the viscous region of the inner flow. At $y / \delta>0.5$, three terms contribute significantly (i.e. $-\langle u v\rangle \approx+40 \%, \mathrm{PG} \approx+80 \%$, convection $\approx-20 \%$ ). It is observed that the streamwise gradients of the Reynolds stresses $\left(\left\langle u^{2}\right\rangle\right.$ and $\left.\left\langle v^{2}\right\rangle\right)$ are negligible everywhere. In the outer part of the boundary layer, the strong pressure gradient is balanced by the mean convection terms. More importantly, the sum of all the terms produces the wall shear stress as shown by the solid line. It is a constant throughout the entire layer as it must be according to (3.1). The value of the wall 

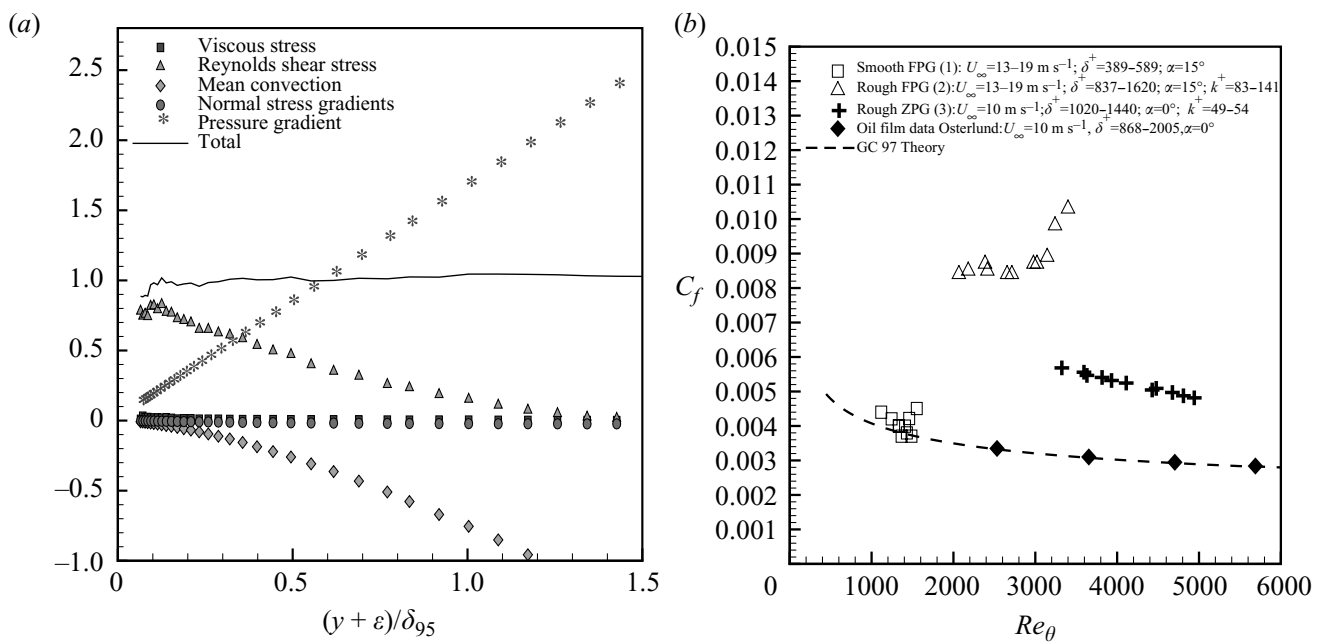

FIGURE 6. Momentum balance calculation and skin friction coefficient. (a) Momentum balance for rough FPG data at $x=1.6 \mathrm{~m}$. (b) Skin friction for FPG and ZPG flows.

shear stress was determined by averaging the straight line data between a value of $0.3<(y+\epsilon) / \delta<1.1$. There is a strong indication that the boundary layer is two-dimensional. If this were not the case, two more mean terms and one turbulence term in the spanwise direction would appear in (3.1); the sum of the terms would be a constant only if these terms were included. Neglecting these terms, as is done here, would have yielded a varying sum if the flow was influenced by secondary flows. Moreover, the deviations from a perfectly straight sum line are within $3 \%$ and can be used as a measure of the uncertainty in the determination of the wall shear stress.

After calculating the wall shear stress for each of the profiles using (3.1), the skin friction $C_{f}$ is plotted against the Reynolds number $R e_{\theta}$ as observed in figure $6(b)$. For comparison purposes and represented by the dashed line, the George \& Castillo (1997) power-law theory for smooth surfaces along with the direct oil film interferometry measurements of Österlund (1999) are included for a $10 \mathrm{~m} \mathrm{~s}^{-1}$ ZPG flow. Some of the smooth FPG data points, those corresponding to x-values close to the leading edge, fall on top of this line, and, as expected, the skin friction values decrease with the Reynolds number. Proceeding further downstream, however, the skin friction values start to increase due to the FPG, and simultaneously the increasingly stronger FPG also causes the Reynolds number to become nearly constant. For this smooth case the viscous drag plays an impontant role, and thus a dependence on $\delta^{+}$and $k^{+}$exists. The skin friction increases significantly as the roughness parameter increases, as observed in figure $6(b)$ for the two rough FPG data sets. For the fully rough FPG data set, Case 2, the skin friction is invariant, and this is also what we observe in figure $6(b)$ up to an $x$-value corresponding to $R e_{\theta} \approx 3000$. However, the skin friction also depends on the FPG and the roughness parameter. Proceeding downstream from this point, the increasing pressure gradient starts to influence the boundary layer development significantly, leading to an increase in the skin friction. This in turn causes the roughness parameter $k^{+}$to increase, and the skin friction increases even more. The FPG also changes the shape of the velocity profile to become 'fuller', and thereby the Reynolds number $R e_{\theta}$ increases only slowly. The fully rough data is mostly composed of form drag since the viscous sublayer is destroyed by the rough surface. As a result, the viscous drag contribution is minimal. This is an observation consistent with the 


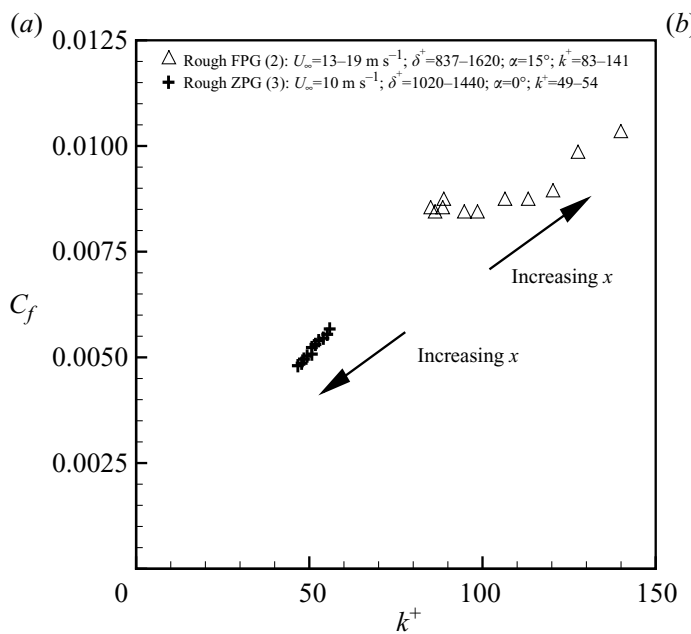

(b)

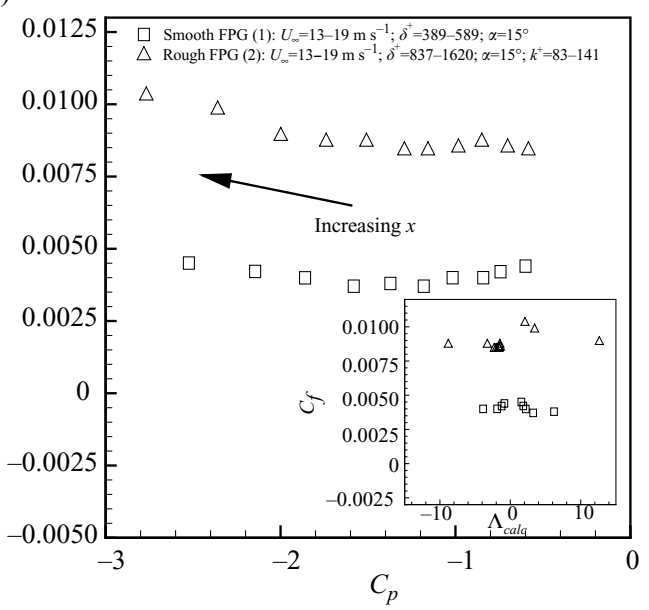

FIGURE 7. Skin friction coefficient dependence on the surface roughness and FPG. $(a) C_{f}$ versus $k^{+} ;(b) C_{f}$ versus $C_{p} \& \Lambda_{\text {calc }}$.

study of Coleman et al. (1977) for rough FPG flows and Brzek et al. (2008) for rough ZPG flows. Given the contribution of so many variables, a function describing $C_{f}=C_{f}\left(R e_{\theta}, k^{+}, \Lambda\right)$ is not yet possible. The skin friction computed using the fully integrated boundary layer equation is within 3-5\% for the ZPG and FPG data sets, respectively.

The skin friction is plotted as a function of both surface roughness and pressure gradient in figure 7. In figure 7(a), for the rough ZPG data documented in Brzek et al. (2007), the skin friction increases monotonically as the roughness parameter is increased. This is expected to be true only for transitionally rough data. The variation in $C_{f}$ of the rough FPG data set is much less than that of the rough ZPG up to $k^{+} \approx 120$. For higher $k^{+}$values, a monotonic increase is observed due to the imposed external FPG. The FPG condition at the last locations is the highest. In figure $7(b)$, the dependence of the skin friction on the FPG condition $\left(C_{p}\right.$ coefficient \& $\left.\Lambda_{\text {calc }}\right)$ is examined. The local value for the pressure parameter $\Lambda_{\text {calc }}$ is plotted in the inset of this figure and is obtained by evaluating $\Lambda$ locally. Similar trends are observed in both figures. There are slight increases in the skin frictions as the pressure gradient strength is increased. The rough FPG values of the skin friction supersede those of the smooth FPG points mainly due to the surface roughness effects. For the rough ZPG data in the transitionally rough regime, the variation in skin friction must occur as a consequence of the roughness parameter.

\section{Results}

The two-dimensional velocity field, including the mean velocity and the Reynolds stresses, is measured at 11 consecutive streamwise locations. The two new data sets presented here together with the rough ZPG case from Brzek et al. (2007) provide clear information about the development of the flows and the influence of the pressure gradient, as well as the surface roughness, on the turbulent boundary layer. These experimental data sets comprise the most comprehensive rough FPG data available in the literature, summing up to 33 different measured profiles. To facilitate clarity, only data from four of the stations are plotted in figures 8-11. A detailed comparison 
at a fixed downstream location $x$ is also performed for the Reynolds stresses. The production terms are investigated in $\S 4.3$.

\subsection{The mean velocity deficit profiles}

The mean velocity deficit profiles are normalized using three different scalings in figure 8 . These scalings are the friction velocity $u_{*}$, free stream velocity $U_{\infty}$ and a scaling, which includes the ratio of $\delta_{*} / \delta$ as proposed by Zagarola \& Smits (1998), $U_{\infty}\left(\delta_{*} / \delta\right)$. Notice that in the limit as $\delta^{+} \rightarrow \infty, \delta_{*} / \delta \rightarrow$ constant for ZPG flows; thus the ZS scaling reduces to the CG scaling. The smooth ZPG data of Castillo \& Johansson (2002) has also been included in the mean velocity deficit profiles.

Figure $8(a)$ shows the mean velocity deficit normalized with the friction velocity. In most literature, the friction velocity is used as the scaling of choice, particularly in rough ZPG flows. It is notable that for the pressure gradient flows, the profiles do not collapse into a single profile, thus showing a Reynolds number dependence for FPG flows. However, the friction velocity absorbs most of the effects of roughness and Reynolds number dependence, while the effects of pressure gradient stand out clearly. The FPG causes the profiles to decrease in magnitude due to the acceleration of the mean flow. The removal of the roughness effects is such that the FPG data falls close to each other, regardless of the roughness parameter range. When comparing the two ZPG cases, it is shown that these are in fairly good agreement showing that the friction velocity removes the effects of roughness from the velocity profiles.

In figure $8(b)$, the mean velocity deficit profiles normalized by $U_{\infty}$ are shown. The velocity profiles in this scaling do not collapse into a single profile, but they are clearly clustered into one set containing the rough profiles, and another one containing the smooth profiles. The collapse of the ZPG data set is better than both of the FPG sets (smooth and rough) for which the profiles show a weak dependence on Reynolds number. In addition, surface roughness also shows its effects on the outer part of the boundary layer. The profiles move upward given an increase on the surface roughness when comparing the smooth and rough FPG data. Even though this boundary layer is subjected to an FPG, the roughness has a larger impact on the mean velocity deficit profiles than the pressure gradient condition. On the outer flow, these two effects counteract each other, where the FPG diminishes the profiles in magnitude, while the surface roughness augments the mean velocity deficit value. However, close to the wall, both promote an increase in the mean deficit velocity profiles given the large values of both $k^{+}$and $k / \delta$. The smooth ZPG data falls in between the smooth FPG and the rough ZPG data thus drawing the conclusion that the roughness increases the magnitude of the velocity deficit while the external pressure gradient decreases it.

Figure $8(c)$ shows the mean velocity deficit data normalized with the $U_{\infty} \delta_{*} / \delta$ scaling. The effects of the roughness and the strength of the pressure gradient appear in the profiles, even though the effects are less notable than when using the free stream velocity or the friction velocity. Surprisingly, on close inspection, the effects of roughness are more notable than those created by the external FPG. This indicates that both the roughness and strength of the pressure gradient could still play a role in the shape of the asymptotic profiles. This may be due to the high ratio of $k / \delta$, where it surpasses the threshold of 0.02 and the lack of an inertial sublayer as suggested by Jiménez (2004). This threshold for the $\bar{k}$ parameter could be lower for FPG flows, since the external FPG reduces the size of the different regions composing the boundary layer; this is also shown by Cal (2006). 

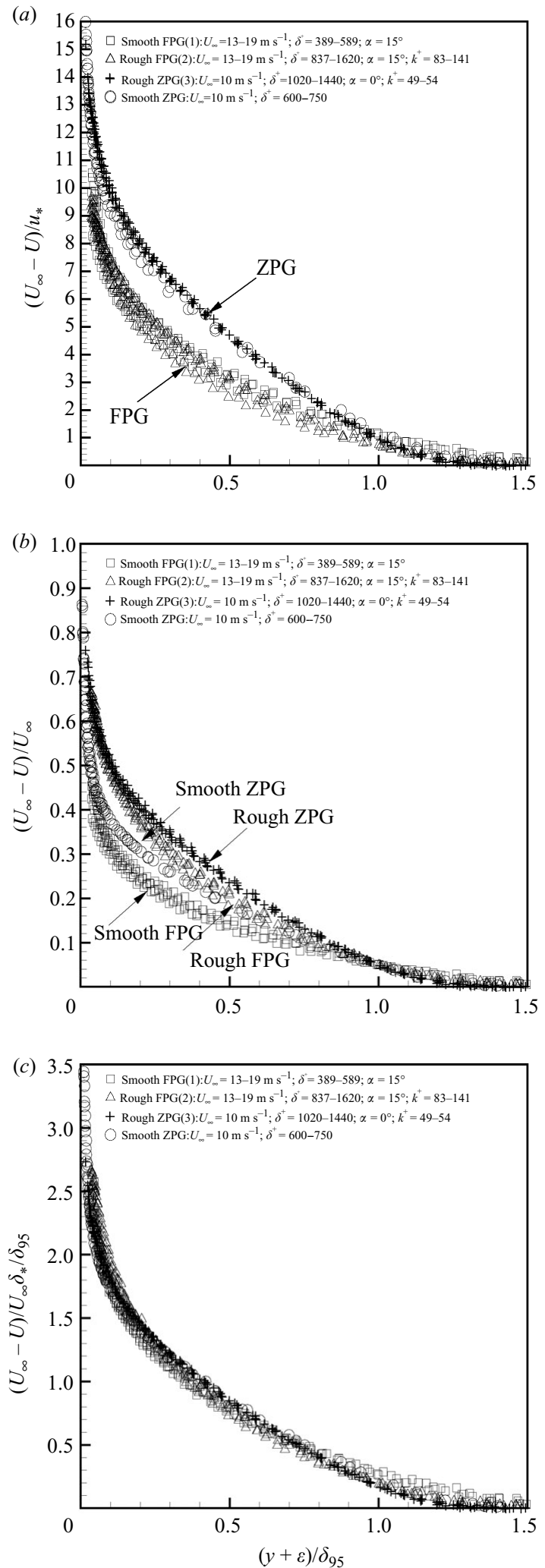

FIGURE 8. Mean deficit velocity profiles for smooth and rough pressure gradient flows: (a) $u_{*}$ scaling, (b) $U_{\infty}$ scaling, (c) $U_{\infty} \delta_{*} / \delta$ scaling. 


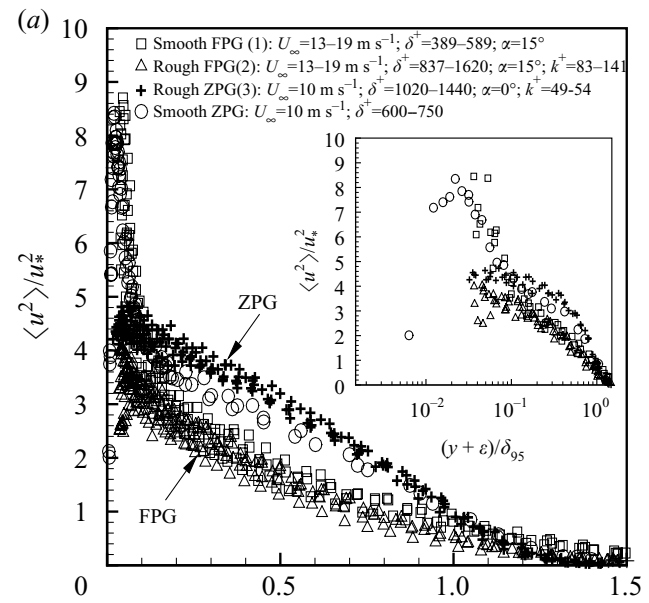

(b)

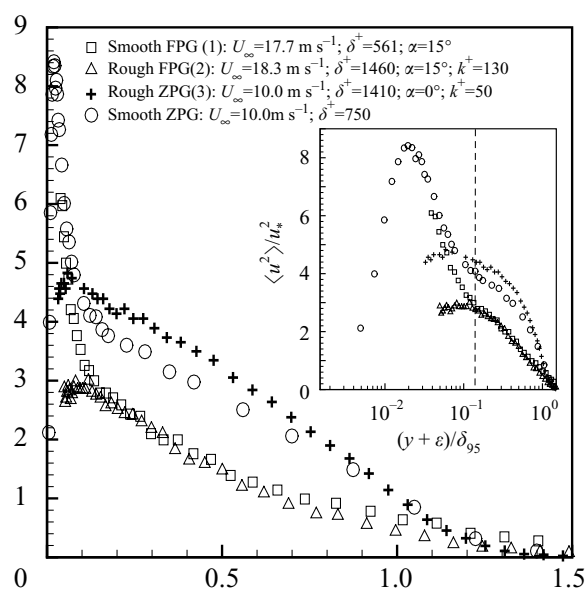

(c)

(d)
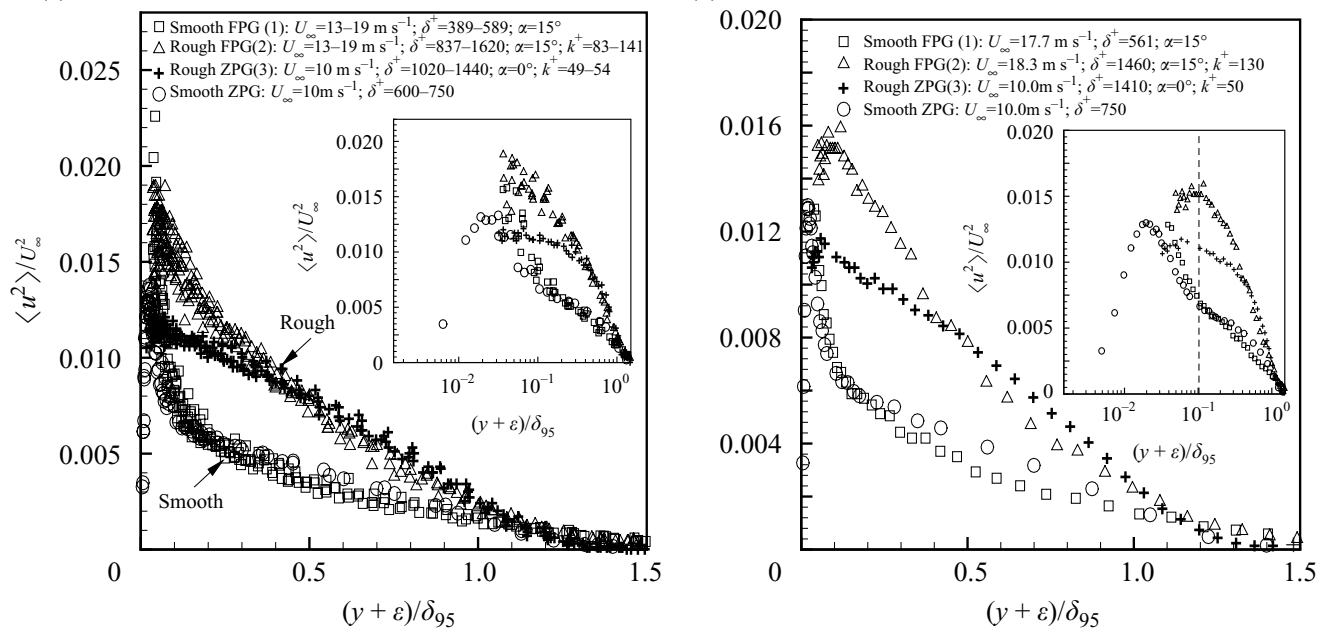

FIGURE 9. Reynolds normal stress $\left\langle u^{2}\right\rangle$ profiles for smooth and rough pressure gradient flows. Fixed position location at $x=2.2 \mathrm{~m}$. (a) $u_{*}^{2}$ scaling, (b) fixed position: $u_{*}^{2}$ scaling, (c) $U_{\infty}^{2}$ scaling, (d) fixed position: $U_{\infty}^{2}$ scaling.

\subsection{The Reynolds stress profiles}

The Reynolds stress components, $\left\langle u^{2}\right\rangle,\left\langle v^{2}\right\rangle$ and $-\langle u v\rangle$ are also measured as part of this study and shown in figures 9-11, respectively. On figures 9-11 $(a \& b)$, the Reynolds stresses are normalized with the friction velocity $u_{*}^{2}$; while figures 9-11 $(c \& d)$ are normalized using the free stream velocity $U_{\infty}^{2}$. On the left-hand side of figures 9-11, four stations of the different cases are plotted, while on the right-hand side of figures 9-11, a fixed location is plotted at $x=2.2 \mathrm{~m}$.

Starting with the streamwise normal component of the Reynolds stress tensor $\left\langle u^{2}\right\rangle$, the friction velocity is employed to normalize the profiles as seen in figure $9(a)$. Here the effects of roughness are nearly removed from the outer region, but the effects caused by the FPG are observed where the magnitude of $\left\langle u^{2}\right\rangle$ diminishes due to the FPG. This behaviour is consistent with that seen by Nickels (2004). There is also a weak Reynolds number dependence on the profiles subject to an FPG. For ZPG 
on smooth/rough surfaces, the $u_{*}$ scaling supports the similarity hypothesis on sand grain type surfaces as proposed by Townsend (1956) and defended by Flack et al. (2005). However on FPG flows subject to roughness, the flows fail to support this hypothesis. Smooth ZPG data is also included for comparison. A slight Reynolds number dependence is observed in this plot where similar trends not only in the dependence but in the shape of the profiles are observed by Morrison et al. (2004) and Jiménez \& Hoyas (2008).

In figure $9(b)$, the $\left\langle u^{2}\right\rangle$ Reynolds stresses are evaluated at a fixed downstream location. The FPG condition alters the overall magnitude by decreasing the data. In the inner region, the smooth data (ZPG and FPG) produces higher values for this component than the rough data. In the outer part, there is an upward shift in the curvature of the smooth FPG profile. This can be attributed to the acceleration due to the pressure gradient caused by the mean flow gradient in the streamwise direction. Meanwhile, the surface roughness produces a smaller effect on the data than the pressure gradient. Although, a Reynolds number dependence in this case may not be ruled out. The structure of the inner layer is altered by the roughness elements destroying the near-wall peak. Therefore, this dampens the magnitude of the stress close to the wall for this component. This reduction in the near wall peak is also seen with the $U_{\infty}^{2}$ scaling. Notice that aside from the inner layer, the outer layer follows a common trend even with the difference in Reynolds number.

When using the free stream velocity, the profiles show both the effects of the rough wall and the imposed FPG as observed in figure $9(c)$. The effect of roughness dominates the boundary layer when comparing the magnitude of its influence on the profiles. The magnitude of the stress is higher for the rough surface than for the smooth surface outside $(y+\epsilon) / \delta_{95}>0.04$. Furthermore, the profiles tend to increase near the wall as a result of the FPG. Conversely, the profiles show a moderate difference due to the strength of the pressure gradient in the outer region. Although a slight increase in magnitude is noticed close to the wall, the external pressure gradient dampens the fluctuations in the outer part of the boundary layers. This argument gives further indication that the roughness effects supersede those caused by the pressure gradient in this scaling. This influence is also schematically observed in figure 3 , whereas the roughness parameter $k^{+}$augments a larger influence which is seen in the different regions of the boundary layer. The shape of the profiles is also different for smooth to rough surfaces, as shown in figure $9(c)$. This observation is emphasized in the insert graph of figure $9(c)$, which is plotted in semilog scale. A deduced observation is that the increase in strength of the pressure gradient leads to a lower value of the Reynolds stress. However, the effect of roughness counteracts the effect of the FPG. Noticing the profiles in semi-log scale, the change in shape over the entire inner and outer regions is evident.

In figure $9(d)$, the data is normalized using the free stream velocity, $U_{\infty}^{2}$ at a fixed downstream location. The imposed acceleration causes an increase close to the wall, while the outer part of the flow is dampened due to the FPG on the streamwise component of the Reynolds stress. Meanwhile, the roughness increases the magnitude of this component in the outer region. The change in the shape is also evident due to both roughness and FPG. Close to the wall in the inset of figure $9(d)$ and plotted in semilog scale, the trend of the streamwise component for the smooth FPG curve is concave up while the rough curves (both ZPG and FPG) are concave down. This change in shape is attributed to the effects produced by the surface roughness, which destroys the viscous sublayer region as the roughness parameter increases as shown by Cal et al. (2008). With this component, the most drastic changes occur 

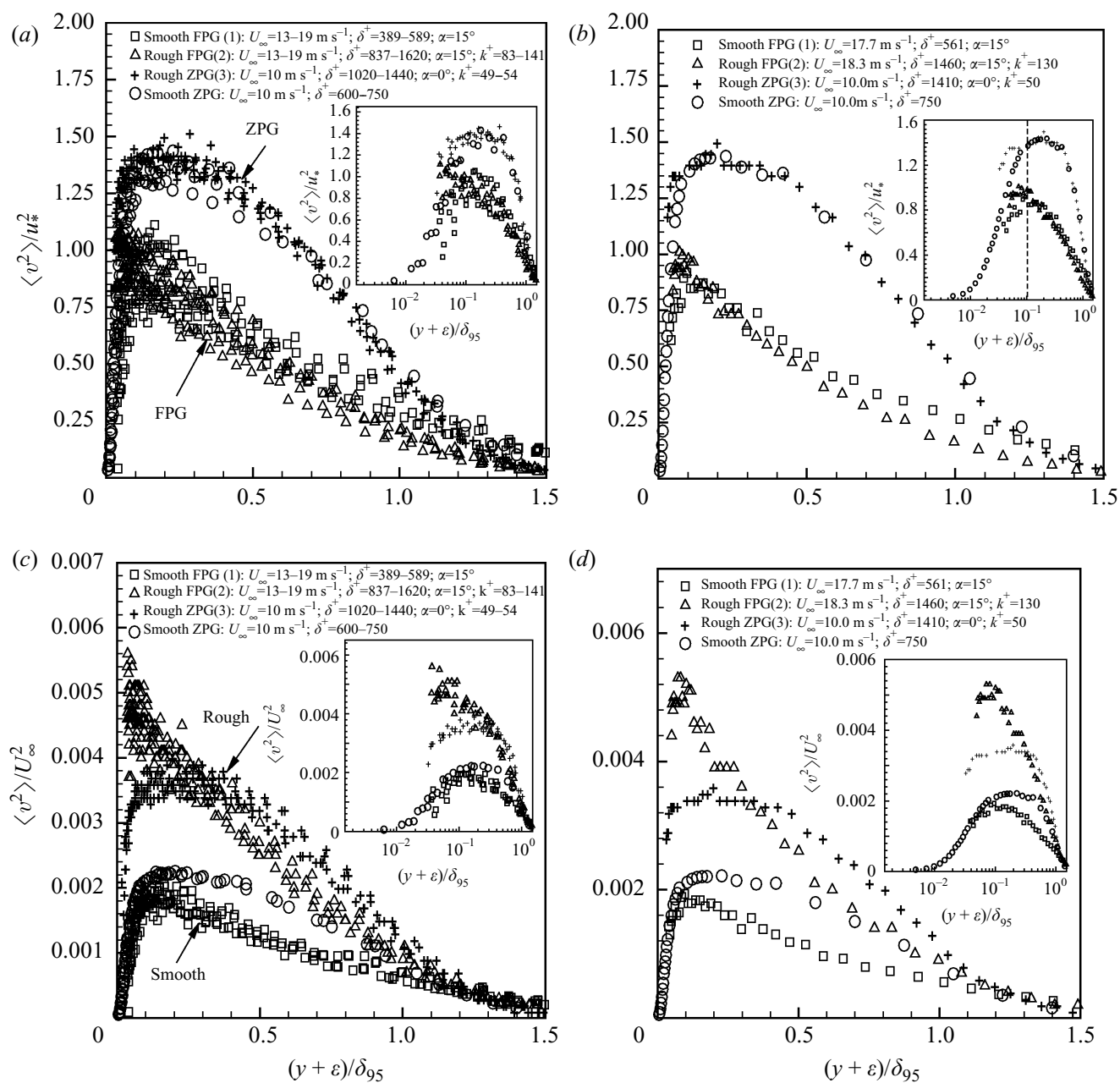

FIGURE 10. Reynolds normal stress $\left\langle v^{2}\right\rangle$ profiles for smooth and rough pressure gradient flows. Fixed position location at $x=2.2 \mathrm{~m}$. (a) $u_{*}^{2}$ scaling, (b) fixed location: $u_{*}^{2}$ scaling, $(c) U_{\infty}^{2}$ scaling, (d) fixed position: $U_{\infty}^{2}$ scaling.

and the magnitude is significantly larger than in the wall-normal and shear stress components.

In the $\left\langle v^{2}\right\rangle$ component of the Reynolds stress, the profiles for the two FPG cases (smooth and rough) normalized with the friction velocity in figure $10(a)$ exhibit dependencies on the local Reynolds number and the increasing FPG strength. However, there is not a significant difference between the smooth and rough data (sets 1 and 2). Near the wall, the difference exists in the shape of the profiles where roughness causes a strong upward shift of the data. Also, roughness causes the ZPG and FPG data, to become 'flatter' as the roughness parameter is increased. The external pressure gradient suppresses the $\left\langle v^{2}\right\rangle$ component throughout the entire layer and a considerable difference between the ZPG and FPG data exists over the entire boundary layer. Differences caused by the rough surface between rough ZPG flows were observed by Bakken et al. (2005) with these discrepancies being attributed to the use of different boundary conditions. 
In figure $10(b)$, the wall-normal Reynolds stress component $\left\langle v^{2}\right\rangle$ is shown at a fixed location and normalized with $u_{*}^{2}$. The behaviour and change in terms of magnitude is the most prominent out of all the Reynolds stress components. The surface roughness destroys the structures in the inner region where the profile becomes 'flatter'. This becomes even more evident for the rough FPG data. Even for the rough ZPG data, there is a dip in the data close to the wall similar to the smooth FPG. As with the streamwise component, the wall-normal component is drastically damped due to the FPG condition. Furthermore, the friction velocity scaling is able to better absorb the effects due to the roughness than those created by the external FPG.

However, when the $\left\langle v^{2}\right\rangle$ component is scaled with the free stream velocity, both effects are evident here, contrary to the observations of the streamwise component of the Reynolds stresses. For example, in figure 10(c), the behaviour of this component changes when comparing the ZPG and FPG flows on a rough wall (sets 2 and 3). The rough FPG data is damped away from the wall $(y / \delta<0.5)$ faster than the rough ZPG data. Furthermore, the shape of the profiles is nearly the same in the outer layer for the smooth and rough wall FPG boundary layers, as seen in figure $10(c)$ despite the large difference in magnitude. The profiles change close to the wall, where the FPG augments the magnitude of the stress, whereas the opposite behaviour is observed in the outer layer. This component exhibits the largest difference in magnitude due to an FPG compared to the $\left\langle u^{2}\right\rangle$ and $-\langle u v\rangle$ components. Also, it is notable that this set of data shows a slight Reynolds number dependence. The current observations are in agreement with the findings of Cal \& Castillo (2008) for flows subject to strong FPGs.

Surprisingly, the shape of the smooth FPG and rough ZPG data sets resemble each other for the $\left\langle v^{2}\right\rangle$ component, as observed in the inset of figure $10(d)$. Here, the behaviour of the profiles are concave down regardless of the roughness or pressure gradient effect for these particular data sets, which is a near-wall behaviour. On the outer layer beyond $\bar{y}>0.1$, the rough ZPG data set is different in shape, which points to an effect caused by the external FPG. Even though this is observed, the magnitudes of the roughness and FPG effects are very different from those appreciated in the $\left\langle u^{2}\right\rangle$ component, as seen in figure $10(d)$.

The fact that the wall-normal component of the Reynolds stress is affected the most by the pressure gradient is a further indication that there is a balance between these two, as seen in the $y$-momentum equation, where $0=-(1 / \rho) \partial P / \partial y-\partial\left\langle v^{2}\right\rangle / \partial y$. The displacement of $\left\langle v^{2}\right\rangle$ close to the wall is due to the FPG, which is caused by the pressure diffusion term $(2\langle p v\rangle)$ of the wall-normal component of the Reynolds stress equation. Furthermore, the production term close to the wall when subject to the pressure gradient is the cause for the prior behaviour, since it does not contribute to the pressure-strain rate (i.e. the redistribution of the energy) on the wall-normal component of the Reynolds stress. Furthermore, as an overall effect, it is observed that the surface roughness effect supersedes that of the FPG in magnitude. It is also important to note the susceptibility of the friction velocity scaling to the FPG. Due to these observed behaviours, it is clear that scaling the Reynolds stress and mean velocity deficit subject to FPG and roughness is a multiple scaling problem; where the scaling themselves need to possess information about the inner (roughness) or outer layer (FPG). In this case, the free stream velocity contains information about the external pressure gradient (i.e. absorbs pressure gradient effects) and the friction velocity contains information related to the wall (i.e. absorbs surface roughness effects).

In figure 11 , the Reynolds shear stress $-\langle u v\rangle$ is normalized by $u_{*}^{2}$ and $U_{\infty}^{2}$. The data normalized with the friction velocity exhibits a similar trend as in the $\left\langle v^{2}\right\rangle$ component, 

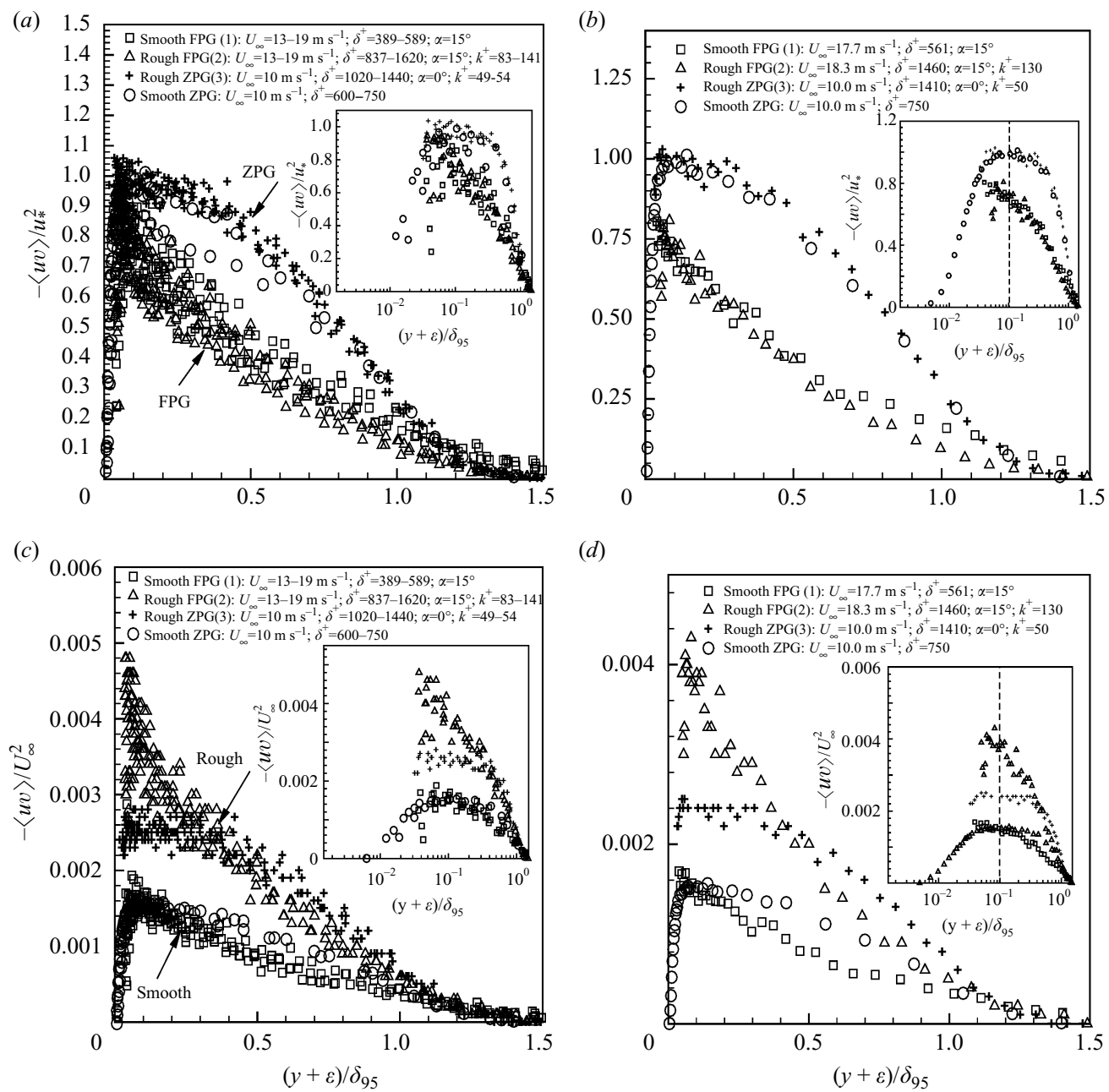

(d)

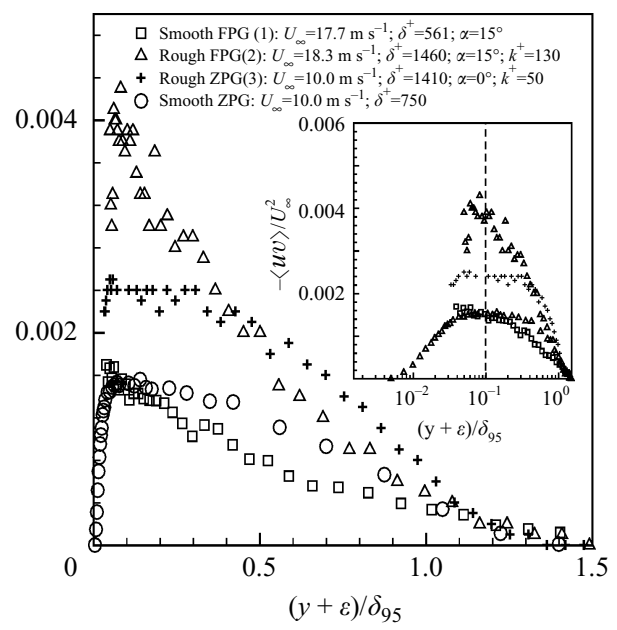

FIGURE 11. Reynolds shear stress $-\langle u v\rangle$ profiles for smooth and rough pressure gradient flows:

(a) $u_{*}^{2}$ scaling, (b) fixed position: $u_{*}^{2}$ scaling, $(c) U_{\infty}^{2}$ scaling, $(d)$ fixed position: $U_{\infty}^{2}$ scaling.

where the major difference is observed when the rough data sets are compared (sets 2 and 3). There is a decrease in the profiles due to the imposed FPG condition, although the change in the behaviour in the $\left\langle v^{2}\right\rangle$ component is more drastic than in this component.

Moreover, the Reynolds shear stress profiles, as shown in figure 11(b), again show the same trends in behaviour as the $\left\langle v^{2}\right\rangle$ component and the magnitudes are also similar, but still do not surpass the effects by the FPG or the surface roughness in the $\left\langle v^{2}\right\rangle$. In terms of the changes in magnitude, again there is a suppression of the $-\langle u v\rangle$ component due to the FPG. On the contrary, a minute difference exists due to the surface roughness. This confirms that the friction velocity scaling possesses information about the wall (i.e. surface roughness). Also, another observation from figure $11(b)$ seen clearly on the insets is the similarity in shape of all the rough profiles. This similarity is due to the effect of surface roughness which aids the flow to become more isotropic in the near-wall region. This finding is consistent with that of Brzek et al. (2007) for ZPG flows. 
The scaling obtained from similarity analysis, $U_{\infty}^{2} \mathrm{~d} \delta / \mathrm{d} x$, is not used here since the boundary layer growth rate $\mathrm{d} \delta / \mathrm{d} x$ becomes negative when the imposed FPG is strong enough. Evidently, the data would not collapse using the scaling obtained through similarity analysis for finite Reynolds number. Therefore, when using $U_{\infty}^{2}$, the same trends as the wall-normal component are apparent. The Reynolds shear stress shows a large shift upwards on the profiles due to the surface roughness as seen in figure $11(c)$, especially near the wall. The effects of the pressure gradient are again noticeable, where close to the wall, the rough FPG data displaces upwards in comparison to the rough ZPG data. Furthermore, it is observed that all of the Reynolds stresses show a slight Reynolds number $\delta^{+}$dependence within the Reynolds number intervals investigated here. The equivalent values in $R_{\theta}$ is 1118 up to about 4927. It is in this range where the greatest variation due to Reynolds number exists in the zero-pressure gradient boundary layer as shown by Castillo (1997). It is also evident that the sand grain roughness alters the shape of the profiles in the inner region by increasing the magnitude of the component all along the boundary layer while the external FPG suppresses the Reynolds stresses.

Figure $11(d)$ shows an increase in magnitude in Reynolds shear stress $-\langle u v\rangle$ component, due to the surface roughness. The shapes of the FPG data sets are similar, contrary to the profiles for the $\left\langle u^{2}\right\rangle$ component. Observing carefully the profiles for the rough FPG and rough ZPG data, the reduction due to the imposed FPG on the $\left\langle v^{2}\right\rangle$ and $-\langle u v\rangle$ components of the Reynolds stress is evident in the outer region. Conversely, there is an increase in the inner region due to the increase in the FPG. This is seen all the way out to the edge of the outer layer. In this case, the general observation when using the square of the free stream velocity as a scaling is that this quantity has information about the flow far from the wall. Thus the effects of pressure gradient are absorbed since this condition is imposed externally. The results point to a possibility of the Reynolds stresses having to be normalized using a mixed scaling. This may be necessary given the different behaviours away and close to the wall depending on the employed scaling, as also suggested by Degraaff \& Eaton (2000) for ZPG flows.

In light of the study conducted by Coleman et al. (1977), it was found that the results are in agreement when both the velocity deficit and Reynolds stress profiles are normalized with the friction velocity. Furthermore, when scaled with the free stream velocity, the $\left\langle u^{2}\right\rangle$ component agrees with their studies. A 'cross-over' behaviour is observed, where close to the wall, there is an increase due to the FPG and away from the wall, the magnitude of the component diminishes. On the other hand, the $\left\langle v^{2}\right\rangle$ component is ill-behaved, where the component is dampened as the FPG is imposed in the study of Coleman et al. (1977). In the present study, the accelerated and unaccelerated cases both possess the same magnitude, although they display the same cross-over nature as in the streamwise component of the Reynolds stress. As a general result, the roughness effects spread over all of the different components, affecting each one equally (same magnitude) when normalized with either scaling, thus promoting isotropy near the wall.

\subsection{The production term}

Given the vast number of measurement locations along the streamwise direction, the $x$-dependence is obtained and the production term is computed. The production term is given by

$$
P_{i j}=-\left\langle u_{i} u_{k}\right\rangle \frac{\partial U_{j}}{\partial x_{k}}-\left\langle u_{j} u_{k}\right\rangle \frac{\partial U_{i}}{\partial x_{k}} .
$$



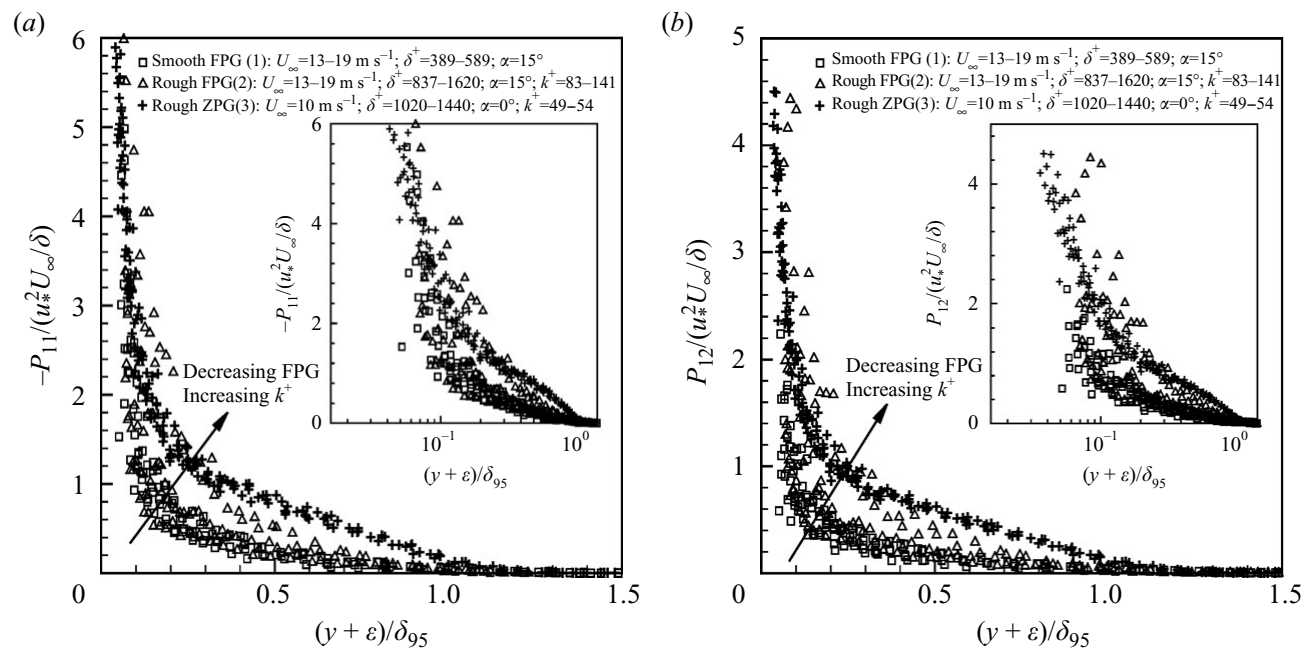

FIgURE 12. Production term $P_{i j}(a)$ production component $P_{11} ;(b)$ production component $P_{12}$.

This quantity is responsible for the production of the Reynolds stress, due to the mean velocity gradients. The $\left(U_{\infty}^{3} / \delta_{95}\right) \mathrm{d} \delta / \mathrm{d} x$ scaling is used to normalize the $P_{11}$ component of the production term while the $P_{12}$ component with $U_{\infty}^{3} / \delta_{95}$ as suggested by George \& Castillo (1997). In this study for comparison purposes, the scaling used for the $P_{11}$ component is $u_{*}^{2} U_{\infty} / \delta_{95}$ which is obtained from matching the Reynolds stress (i.e. $\left.u_{*}^{2} \sim U_{\infty}^{2}\left(\mathrm{~d} \delta_{95} / \mathrm{d} x\right)\right)$. It is important to note that the terms which possess the $x$-dependence are usually neglected (i.e. $\partial U / \partial x, \partial V / \partial x$ ), since normally not more than one downstream location is measured. However, the $x$-dependence is negligible compared to the wall normal gradients for ZPG flows.

The $P_{11}$ component of production is shown in figure 12(a) normalized with $u_{*}^{2} U_{\infty} / \delta_{95}$. The curves for both rough cases fall almost on top of each other, but if analysed carefully, in the outer part of the boundary layer, the production of the rough ZPG is higher than the rough FPG. As a consequence, this reinforces the point that the FPG suppresses the turbulence production as observed in the Reynolds stress profiles regardless of the employed scaling. The common trend is that the production of turbulence with this component is decreased as the external FPG is increased. In terms of the roughness effect, it is obvious that the rough surface promotes turbulence. However, this is consistent with the increase in the Reynolds stresses observed in $\S 4.2$.

The production of the Reynolds shear stress $P_{12}$ displays the same behaviour as $P_{11}$ as shown in figure $12(b)$. Moreover, the profiles increase marginally in magnitude as the strength of the FPG is decreased. This means that for this component, the roughness enhances the production of the turbulence significantly, while the pressure gradient diminishes it as observed in the Reynolds stress profiles. It is also observed that both components $P_{11}$ and $P_{12}$ are of the same order of magnitude.

In the wall-normal and spanwise Reynolds stress equations, there are no production terms present. This provides further evidence that the production in these two directions (wall-normal and spanwise) is 'injected' by the redistribution of energy caused by the pressure-strain rate terms. Following this statement, the pressure diffusion terms decrease due to the acceleration produced by the FPG, since the production acts against the pressure-strain terms (i.e. opposite signs of production and pressure strain). The same is deduced for the dissipation of turbulence. This 
means that the flow becomes less dissipative due to the imposed FPG on the flow. It also damps turbulence on the outer layer, while enhancing it close to the wall. On the other hand, the surface roughness enhances the production of turbulence. This acts 'negatively' with the pressure-strain rate and the opposite with dissipation. Furthermore, the surface roughness then increases the exchange of energy among components through the pressure-strain rate, and also increases the dissipation of the flow. As a consequence, there is a balance between the FPG and the surface roughness. In general, the effect of the surface roughness is higher than the FPG in terms of magnitude. These observations support those reported by Coleman et al. (1977).

\section{Conclusions}

LDA measurements on a two-dimensional flow are carried out over 11 consecutive streamwise locations for three experimental sets yielding 33 profiles. Consequently, the $x$-dependence of the boundary layer is reported in this study from which the wall shear stress is extracted including the streamwise gradients of the flow. This is the first experiment of its kind using LDA on rough FPG turbulent boundary layers. The outcomes of this study are four-fold: (i) calculated the skin friction independently using the full integrated momentum equation; (ii) performed experiments to sort out the differences due to the FPG and the surface roughness effects; (iii) isolated surface roughness and FPG effects on the outer flow by testing the $u_{*}, U_{\infty}$ and $U_{\infty} \delta_{*} / \delta$ mean velocity scalings and the $u_{*}^{2}$ and $U_{\infty}^{2}$ scalings for the Reynolds stresses; (iv) studied flow parameters such as the $\delta_{*} / \delta$ ratio, $\Lambda$, as well as, the production to understand the influence of the FPG and surface roughness.

The wall shear stress $\tau_{w} / \rho$ is obtained using the full integrated momentum equation, thus including the streamwise gradients of the two-dimensional velocity field. In the proximity of the wall, the Reynolds shear stress and the FPG are the dominating terms contributing $80 \%$ and $20 \%$, respectively. In the intermediate distances, the $-\langle u v\rangle$ and the pressure gradient contribution cross each other and are balanced by the negative mean convective terms, which represent $+40 \%,+80 \%$ and $-20 \%$, respectively. The outer flow is then consummated by the mean convection and the FPG. The viscous terms and the streamwise gradients of the Reynolds normal stresses $\left(\left\langle u^{2}\right\rangle\right.$ and $\left.\left\langle v^{2}\right\rangle\right)$ are negligible throughout the layer with a combined contribution of about $2 \%$. The $C_{f}$ coefficient for the rough FPG is no longer Reynolds number invariant, although the flow is in the fully rough regime. In the fully rough regime, the skin friction is composed solely of form drag. Furthermore, the skin friction coefficient values tend to increase drastically as the strength of the FPG increases. The same trend is observed with increasing $k^{+}$. From the full integrated boundary layer equation, the skin friction was obtained within $3 \%$ and $5 \%$ error for ZPG and FPG data, respectively.

The most notable results related to the velocity field are as follows: the mean velocity deficit profiles normalized by the friction velocity removes the roughness effects in the outer region of the boundary layer, and the $U_{\infty}$ scaling is more effective in absorbing the FPG effects. However, the ZS scaling improves the collapse of the data, but residual effects due to the strength of the pressure gradient are observed in the outer region.

The Reynolds stresses clearly show the effects of the surface roughness and the imposed FPG in this study when normalized with the $u_{*}^{2}$ or $U_{\infty}^{2}$ scalings. The profiles of the streamwise component of the Reynolds stress changes shape entirely due to the roughness imposed at the wall and the strength of the external FPG. Although there 
is an increase in the magnitude of the $\left\langle v^{2}\right\rangle$ component, the shape for the rough FPG resembles that of the smooth ZPG data. The profiles also become flatter due to the influence of the roughness parameter close to the wall, which destroys the coherent structures in the inner layer. This is also observed for the $\left\langle u^{2}\right\rangle$ and $-\langle u v\rangle$ components. The high peak close to the wall and damping away from the wall are due to the pressure diffusion term $\langle p v\rangle$ and the balance of the pressure on the $y$-momentum equation, respectively. The roughness effect is a more uniform effect, increasing all of the Reynolds stresses when scaled with $U_{\infty}^{2}$, thus giving evidence that roughness promotes isotropy close to the wall as in ZPG flows. The variation of the FPG affects the wall-normal and shear components of the Reynolds stress the most when scaled with the $U_{\infty}^{2}$. The streamwise component of the Reynolds stress is affected by the pressure gradient; thus this effect promotes anisotropy on the Reynolds stresses. Moreover, the friction velocity scaling is more susceptible to the pressure gradient condition rather than the surface roughness, while the free stream velocity shows the reverse observation. These observations give further indication that a mixed scaling should be employed since the friction velocity possesses information about the nearwall behaviour, while the free stream velocity contains properties which occur far away from the wall.

The $\delta_{*} / \delta$ ratio also displays such effects caused by the rough surface and the pressure gradient. An increase in pressure gradient causes this ratio to increase. The rough-surface condition not only increases the magnitude of the ratio but also removes the Reynolds number dependence on the parameter. The roughness parameter does not manifest its effects on the pressure parameter, $\Lambda$, and the boundary layer is clearly in non-equilibrium (i.e. since $\Lambda \neq$ constant). Moreover, the boundary layer growth indicates the influence of the development of the turbulent boundary layer due to the roughness and pressure gradient, where the roughness thickens the boundary layer while the FPG suppresses its growth. An increase in surface roughness induces an increase in production of turbulence while an increase in FPG decreases it.

This particular study provides ample insight on the behaviour of the rough FPG turbulent boundary layer by studying different important quantities. These quantities become part of the interaction between the different terms in the momentum and Reynolds stress equations, thus taking into consideration the relationship of these two effects of FPG and surface roughness. This allows for the improvement of the quality of turbulent models and provides an extensive database available for the community.

This work was funded by grants from the Office of Naval Research (ONR) and the National Science Foundation (NSF) - AGEP Program. The authors want to recognize and express their gratitude to programme managers, Dr Ronald Joslin (ONR) and Dr Roosevelt Johnson (NSF), for their continued support. We also want to recognize Mrs Julie Brzek for her input on the manuscript.

\section{REFERENCES}

Akinlade, O. G., Bergstrom, D. J., Tachie, M. F. \& Castillo, L. 2004 Outer flow scaling of smooth and rough wall turbulent boundary layers. Exp. Fluids 37, 604-612.

Bakken, O. M., Krogstad, P.-Å., Ashrafian, A. \& Andersson, H. 2005 Reynolds number effects in the outer layer of the turbulent flow in a channel with rough walls. Phys. Fluids 17, 065101.

Bergstrom, D. J., Kotey, N. A. \& Tachie, M. F. 2002 The effects of surface roughness on the mean velocity profile in a turbulent boundary layer. ASME Trans. J. Fluids Engng 124, 664-670. 
Bradshaw, P. 2000 A note on 'critical roughness height' and 'transitional roughness'. Phys. Fluids 12, 1611.

Brzek, B., Cal, R. B., Johansson, T. G. \& Castillo, L. 2007 Inner and outer scalings in rough surface zero pressure gradient turbulent boundary layers. Phys. Fluids 19, 065101.

Brzek, B., Cal, R. B., Johansson, T. G. \& Castillo, L. 2008 Transitionally rough zero pressure gradient turbulent boundary layers. Exp. Fluids 44 (1), 115-124.

CAL, R. B. 2006 The favourable pressure gradient turbulent boundary layer. PhD dissertation, Rensselaer Polytechnic Institute, Troy, NY.

Cal, R. B., Brzek, B., Johansson, T. G. \& Castillo, L. 2008 Influence of external conditions on transitionally rough favourable pressure gradient turbulent boundary layers. J. Turbul. 9 (38), $1-22$.

CAL, R. B. \& CASTiLlo, L. 2008 Similarity analysis on favourable pressure gradient turbulent boundary layers with eventual quasilaminarization. Phys. Fluids 20, 105106.

Cal, R. B., Johansson, T. G. \& Castillo, L. 2006 The effects of upstream conditions on turbulent boundary layers subject to favourable pressure gradients. AIAA J. 44 (11), 2488-2499.

CAstillo, L. 1997 Similarity analysis of turbulent boundary layers. Ph.D. dissertation, State University of New York, Buffalo, NY.

Castillo, L. \& George, W. K. 2001 Similarity analysis for turbulent boundary layer with pressure gradient: outer flow. AIAA J. 39 (1), 41-47.

Castillo, L. \& Johansson, T. G. 2002 The effects of the upstream conditions on a low Reynolds number turbulent boundary layer with zero pressure gradient. J. Turbul. 3, 031.

Castillo, L., Seo, J., Hangan, H. \& Johansson, T. G. 2004a Smooth and rough turbulent boundary layers at high Reynolds number. Exp. Fluids 36, 759-774.

Castillo, L. \& Walker, D. 2002 The effects of the upstream conditions on turbulent boundary layers. AIAA J. 40 (12), 2540-2542.

CASTro, I. 2007 Rough-wall boundary layers: mean flow universality. J. Fluid Mech. 585, 469-485.

Clauser, F. H. 1954 Turbulent boundary layers in adverse pressure gradients. J. Aeronaut. Sci. 21, 91-108.

Coleman, H. W., Moffat, R. J. \& Kays, W. M. 1977 The accelerated fully rough turbulent boundary layer. J. Fluid Mech. 82, 507-528.

Coles, D. E. 1962 The turbulent boundary layers in a compressible fluid. Tech. Rep. USAF RAND Corp. Rept. R-403-PR. (See also: (1964) Phys. Fluids 7: 1403-1423).

DegrafF, D. \& Eaton, J. 2000 Reynolds-number scaling of the flat plate turbulent boundary layer. J. Fluid Mech. 422, 319-346.

Fernholz, H. H. \& Warnack, D. 1998 The effects of a favourable pressure gradient and of the Reynolds number on an incompressible axissymmetric turbulent boundary layer. Part 1 . The turbulent boundary layer. J. Fluid Mech. 359, 329-356.

Flack, K. A., Schultz, M. P. \& Connelly, J. S. 2007 Examination of a critical roughness height for boundary layer similarity. Phys. Fluids 19, 095104.

Flack, K. A., Schultz, M. P. \& Shapiro, T. A. 2005 Experimental support for Townsend's Reynolds number similarity hypothesis on rough walls. Phys. Fluids 17, 035102.

George, W. K. 1994 Some new ideas for similarity of turbulent shear flows. In Turbulence, Heat and Mass Transfer I, (ed. K. Hanjalic \& J. C. F. Pereira), pp. 13-24, Begell House, NY (ISBN 1-56700-040-1).

George, W. K. \& Castillo, L. 1997 Zero-pressure gradient turbulent boundary layer. Appl. Mech. Rev. $50(11,1), 689-729$.

Herring, H. J. \& Norbury, J. F. 1966 Some experiments on equilibrium turbulent boundary layers in favourable pressure gradients. J. Fluid Mech. 27, 541-549.

ICHIMIYA, M., NAKAMURA, I. \& YAMASHITA, S. 1998 Properties of a relaminarizing turbulent boundary layer under a favourable pressure gradient. Exp. Therm. Fluid Sci. 17, 37-48.

JimÉNEZ, J. 2004 Turbulent flows over rough walls. Annu. Rev. Fluid Mech. 36, 173-196.

JimÉNEZ, J. \& HoYAS, S. 2008 Turbulent fluctuations above the buffer layer of wall-bounded flows. J. Fluid Mech. 611, 215-236.

Jones, M. B., Marusic, I. \& Perry, A. E. 2001 Evolution and structure of sink-flow turbulent boundary layers. J. Fluid Mech. 428, 1-27. 
KARLSSON, R. I. 1980 Studies of skin friction in turbulent boundary layers on smooth and rough walls. PhD thesis, Chalmers University of Technology, Göteborg, Sweden.

Kline, S. J., Reynolds, W. C., Schraub, F. A. \& Runstadler, P. W. 1967 The structure of turbulent boundary layers. J. Fluid Mech. 30, 741-773.

Krogstad, P.-Å. \& Antonia, R. A. 1994 Structure of turbulent boundary layers on smooth and rough walls. J. Fluid Mech. 277, 1-21.

Krogstad, P.-Å. \& Antonia, R. A. 1999 Surface roughness effects in turbulent boundary layers. Exp. Fluids 27, 450-460.

Krogstad, P.-Å., Antonia, R. A. \& Browne, L. W. B. 1992 Comparison between rough- and smooth-wall turbulent boundary layers. J. Fluid Mech. 245, 599-617.

LAUNDER, B. E. 1964 Laminarization of the turbulent boundary layer in a severe acceleration. Tech. Rep. Rep. 77. MIT Gas Turbine Lab. Cambridge, MA, USA.

Leonardi, S., Orlandi, P., Smalley, R. J., Djenidi, L. \& Antonia, R. A. 2003 Direct numerical simulations of turbulent channel flow with transverse square bars on one wall. J. Fluid Mech. 491, 229-238.

Ligrani, P. M. \& MofFat, R. J. 1986 Structure of transitionally rough and fully rough turbulent boundary layers. J. Fluid Mech. 162, 69-98.

Ludweig, H. \& Tillman, W. 1950 Investigations of the wall shearing stress in turbulent boundary layers. Tech. Rep. TM 1285. NACA

Morrison, J., McKeon, B., JiAng, W. \& SMits, A. 2004 Scaling of the streamwise velocity component in turbulent pipe flow. J. Fluid Mech. 508, 99-131.

Mukund, R., Viswanath, P. R., Narasimha, R., Prabhu, A. \& Crouch, J. D. 2006 Relaminarization in highly favourable pressure gradient on a convex surface. J. Fluid Mech. 566, 97-115.

NARAsimha, R. \& SREEnivasan, K. R. 1973 Relaminarization in highly accelerated turbulent boundary layers. J. Fluid Mech. 61, 417-447.

Newhall, K., Cal, R. B., Brzek, B., Johansson, T. G. \& Castillo, L. 2006 Determination of skin friction on smooth and rough turbulent boundary layers subject to external favourable pressure gradients. In ASME Joint U.S.-European Fluids Engineering Conference, FEDSM2006-98517, Miami, FL.

NiCKELS, T. B. 2004 Inner scaling for wall-bounded flows subject to large pressure gradients. J. Fluid Mech. 521, 217-239.

Nikuradse, J. 1933 Laws of Flows in rough pipes, NACA Technical memorandum 1292.

ÖsTERLUND, J. 1999 Experimental studies of zero pressure-gradient turbulent boundary layer flow. $\mathrm{PhD}$ thesis, Royal Institute of Technology, Stockholm, Sweden.

Patel, V. C. \& Head, M. R. 1968 Reversion of turbulent to laminar flow. J. Fluid Mech. 23, 185-208.

Perry, A. E. \& LI, J. D. 1990 Experimental support for the attached-eddy hypothesis in zero-pressure gradient turbulent boundary layers. J. Fluid Mech. 218, 405-438.

Piomelli, U., Balaras, E. \& Pascarelli, A. 2000 Turbulent structures in accelerating boundary layers. J. Turbul. 1, 001.

Radhakrishnana, S., Keating, A. \& Piomelli, U. 2006 Large eddy simulations of high Reynolds number flow over a contoured ramp. In AIAA-2006-0899, 44th AIAA Aerospace Science Meeting and Exhibit, Reno, NV.

Raupach, M. R., Antonia, R. A., \& Rajagopalan, S. 1991 Rough-wall turbulent boundary layer. Appl. Mech. Rev. 44, 1-25.

SchulTz, M. P. 2002 The relationship between frictional resistance and roughness for surfaces smoothened by sanding. ASME J. Fluids Engng 124, 492-499.

Schultz, M. P. \& FlaCK, K. A. 2005 Outer layer similarity in fully rough turbulent boundary layer. Exp. Fluids 38, 324-340.

SEO, J. 2003 Investigation of the upstream conditions and surface roughness in turbulent boundary layers. PhD dissertation, Rensselaer Polytechnic Institute, Troy, NY.

Shafi, H. S. \& Antonia, R. A. 1995 Anisotropy of the Reynolds stresses in a turbulent boundary layer on a rough wall. Exp. Fluids 18, 213-215.

Sreenivasan, K. R. 1982 Laminarescent, relaminarizing and retransitional flows. Acta Mech. 44, $1-48$. 
Tachie, M. F., Bergstrom, D. J. \& Balachandar, R. 2000 Rough wall turbulent boundary layers in shallow open channel flow. J. Fluids Engng 122, 533-541.

Townsend, A. A. 1956 The Structure of Turbulent Shear Flows. Cambridge University Press.

Warnack, D. \& Fernholz, H. H. 1998 The effects of a favourable pressure gradient and of the Reynolds number on an incompressible axissymmetric turbulent boundary layer. Part 2 . The boundary layer with relaminarization. J. Fluid Mech. 359, 357-381.

Zagarola, M. V. \& Smits, A. J. 1998 Mean-flow scaling of turbulent pipe flow. J. Fluid Mech. 373, 33-79. 